\title{
DESIGN AND TEST OF AN ADJUSTABLE QUASI-ZERO STIFFNESS DEVICE AND ITS USE TO SUSPEND MASSES ON A MULTI-MODAL STRUCTURE
}

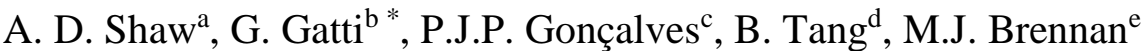

${ }^{a}$ College of Engineering, Swansea University, Swansea, UK

${ }^{b}$ Department of Mechanical, Energy and Management Engineering, University of Calabria, Rende, Italy

${ }^{c}$ Department of Mechanical Engineering, Faculty of Engineering, UNESP, Bauru, Brazil

${ }^{d}$ Key Laboratory of Ocean Energy Utilization and Energy Conservation of Ministry of Education, Dalian University of Technology, Dalian, China

${ }^{e}$ Department of Mechanical Engineering, Faculty of Engineering, UNESP, Ilha Solteira, Brazil

* Corresponding author: gianluca.gatti@unical.it

No. of figures: $\quad 17$

No. of animations 1

No. of tables: $\quad 2$ 


\begin{abstract}
In some applications, such as ground vibration testing in the aerospace industry, it is of interest to observe the modal behaviour of a slender structure while it is statically loaded. One way of statically loading such a structure is to suspend masses using very soft springs. If the springs are linear, then this results in an extremely large static deflection of the springs. This problem could be overcome by dynamically isolating the masses using quasi-zero stiffness (QZS) springs. This paper describes the design, construction and experimental testing of a device that can exhibit QZS. A novel design is proposed that allows the stiffness and the symmetry of the device to be adjusted independently using separate adjustment mechanisms. Quasi-static and dynamic testing of the device show that it can be adjusted to have an extremely low stiffness within the limits of measurement. The main trend of the forcedisplacement curve showed that it had a cubic stiffness characteristic, and that friction was responsible for its hysteretic behaviour. Dynamic testing showed that the device locked-up due to friction at low frequencies, but at high frequencies the device acted as an efficient linear isolator. An experiment was also performed where a mass was suspended on a multimodal beam structure via the QZS device. It was shown that a static load could be applied to the beam without the attached mass appreciably affecting the dynamic response of the beam, despite the suspended mass was about $12 \%$ of that of the host structure.
\end{abstract}

Keywords: Quasi-Zero Stiffness; High-Static-Low-Dynamic-Stiffness; Nonlinear vibration; Vibration absorber. 


\section{INTRODUCTION AND MOTIVATION}

Since the book on quasi-zero stiffness (QZS) by Alabuzhev et al. [1] in 1989, there has been an enormous amount of research carried out on the design and application of devices incorporating QZS. These are nonlinear springs that have the attractive property of having a high static stiffness and a very low dynamic stiffness, so-called HSLDS devices. Some common applications for these devices include vibration isolation, vibration absorbers and vibration energy harvesters [2]. Perhaps the most widespread use of nonlinear stiffness elements has been in vibration isolation [3-11], but they are also being considered for inclusion in metamaterials to adjust band gap performance [12], and similar principles are also exploited in energy harvesting [13]. However, in this paper, the application of interest is in static loading a structure using masses suspended from the structure using QZS devices. When a mass is resiliently attached to a structure through a nonlinear stiffness, a nonlinear vibration absorber is formed, and this is an area where much work has been done, so it is appropriate to first review this work in the context of this paper.

A mass together with a QZS spring can be exploited to provide broadband vibration reduction as a nonlinear energy sink (NES). This was pioneered by Vakakis et al. [14,15], who showed that energy could be efficiently transferred from the host structure to the absorber - the socalled targeted energy transfer approach. Gendelman et al. [16-18] further discussed the attractors of a linear oscillator with an attached nonlinear energy sink under external harmonic excitation, and recently, specific tuning approaches for nonlinear absorbers have been discussed by Viguié and Kerschen [19], and Brennan and Gatti [20]. Yang et al. [21] investigated the performance of nonlinear absorbers attached to a nonlinear host system using power flow analysis. Detroux et al. [22] analysed the performance of the nonlinear tuned vibration absorber, and Habib et al. [23] proposed a design strategy for the absorber. Tang et al. [24] experimentally investigated the characteristics of a nonlinear vibration absorber, and its fundamental performance was further explored in [25].

Although there are some similarities between the static loading of a structure using suspended masses and the attachment of vibration absorbers, the objectives are very different. The aim of static loading a structure during laboratory-based vibration testing is to apply static forces 
that the structure may experience in operation. If this is achieved by adding masses, then the dynamics of the structure will be affected, which is clearly undesirable. This can be avoided if very soft springs are used to suspend the masses, and QZS springs could be ideal for this purpose. Such an arrangement offers a way of observing the modes of a structure while it is subject to static loading, avoiding a shift in its natural frequencies, and has been particularly important to the aerospace industry. Distributed loads such as aerodynamic pressure could be simulated by attaching a large number of small masses to the host structure, each of which is attached using a QZS device. Researchers often transform dynamic loads to static loads with dynamic factors [26] and perform structural optimization of airplane wings [26-29]. For aerodynamic experiments and analysis of wings, the effect of large static pre-flutter deformation should be considered [30,31], and a static load taken into account [32], without affecting the dynamic characteristics of the aircraft wings. To realize a suitable ground vibration test of a highly flexible aircraft, Chang and Hodges [33] developed a multiple-beam model restrained by a bungee cord system with very low stiffness. To simulate the free-free boundary conditions of flexible space structures and to counteract gravity loads in ground vibration testing, Woodard and Housner [27] proposed a zero-spring-rate mechanism and characterize the nonlinear behaviour due to structure imperfections. Yang et al. [34] developed an adaptive feed-forward control scheme to adjust the inertial effects of a zerostiffness suspension system featuring a noncircular disk, which was used as an application in counteracting gravity during ground dynamic testing.

The practical design of a true QZS spring is highly challenging as it is inherently susceptible to parameter errors - the two primary sources of parameter error are stiffness error and static load error [35-38]. Stiffness error occurs because of the need to reduce stiffness to the order of typical measurement errors in relation to the static stiffness of the device. This is due to the natural frequency being proportional to the square root of stiffness. For example, reducing the natural frequency by a factor of 10 requires a stiffness reduction by a factor of 100 . This means that typical errors can lead to much higher natural frequencies than expected, or even negative stiffness leading to complex inter-well responses [39]. Some authors have considered ways to correct stiffness error, for example $[38,40]$. Static load (or payload) error results from the need to exactly locate the system equilibrium point at the point of minimum stiffness, and is again exaggerated in very low stiffness devices. This is because if the equilibrium stiffness is very low, a small error in the estimate of the payload weight (or equivalently the supporting spring stiffness) results in a large displacement of the payload 
away from the optimal point, again resulting in significantly higher than expected stiffness. These two forms of error highlight the need for a QZS device to allow precise adjustment of both stiffness and static load bearing to function successfully. Zhou and Liu presented a prototype of HSLDS spring exploiting magnetism to allow a semi-active control of its stiffness [41]. Le and Nguyen presented a theoretical design that allows for both stiffness and load error correction [42]. In [43] Wang et al. create a magnetic QZS device that allows payload adjustment to isolate a neonatal incubator, and this achieved natural frequencies nearly as low as $1 \mathrm{~Hz}$ for a payload in the range of $2.2 \mathrm{~kg}$ to $2.8 \mathrm{~kg}$. Some further applications of QZS design solution, in the form of an HSLDS isolator are discussed in [39,42,44,45].

The aim of this paper is to design and test a simple mechanical device, that provides true QZS behaviour, for the application of suspending masses on a multi-modal structure to provide static loading. This is achieved by incorporating two adjusters that allow both stiffness error and static load error to be corrected independently. It is shown experimentally that the device achieves zero stiffness within the limits of measurement, and can be exploited to apply a static load to a multi-modal structure without significantly shifting its natural frequencies. The contributions of this paper are thus the design, and static and dynamic testing of a simple and compact mechanical QZS device which has two independent tuning mechanisms for stiffness and payload adjustment. Furthermore, the device is used to demonstrate that a static load can be applied by way of adding a mass to a structure with minimal perturbation of its modal properties.

The paper is organised as follows. Following this introduction, Section 2 describes the fundamental effects of adding suspended masses on a multi-modal structure by using elastic elements. The design of a true QZS device is described in Section 3, and in Section 4 static and dynamic experimental characterisation of the device is carried out. The dynamic behaviour of a simple test-rig in which the QZS device is coupled to a simple cantilever beam is experimentally investigated in Section 5. The paper is concluded with Section 6.

\section{EFFECTS OF ADDING SUSPENDED MASSES ON A MULTI- MODAL STRUCTURE}


In this section, an investigation is carried out into the dynamic behaviour of a multi-modal structure when masses are attached through spring-damper suspension devices. A beam is used as a benchmark for the host structure to illustrate the general effects. Of particular interest is the limiting case when QZS devices are used.

The structure of interest consists of a Euler-Bernoulli cantilever beam with an arbitrary number of suspended masses, excited by a harmonic force with amplitude $F$, as shown in Fig. 1.

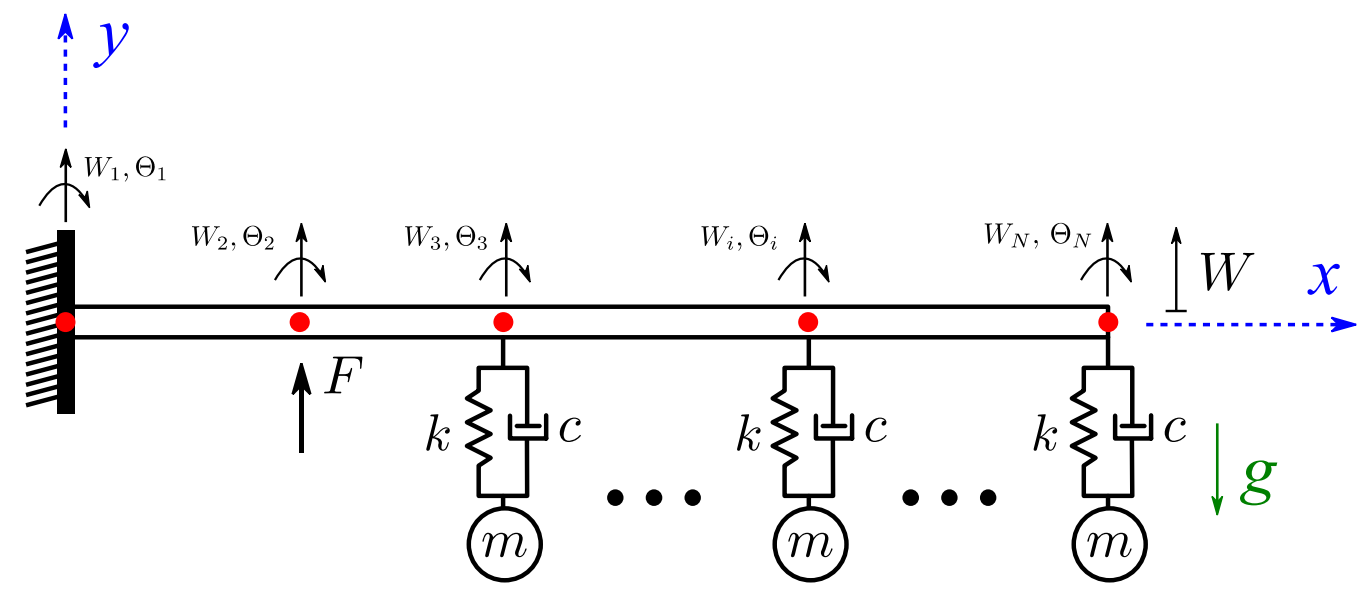

Figure 1. A cantilever beam with an arbitrary number of masses attached through QZS elements and viscous dampers. The g-vector denotes gravity.

The combined system is modelled in the frequency domain using the dynamic stiffness method. A generic beam element of length $L_{e}$ represented in local coordinates, with forces $F_{1,2}$, moments $M_{1,2}$, lateral displacements $W_{1,2}$ and rotations $\Theta_{1,2}$ at each end of the beam element, is shown in Fig. 2.

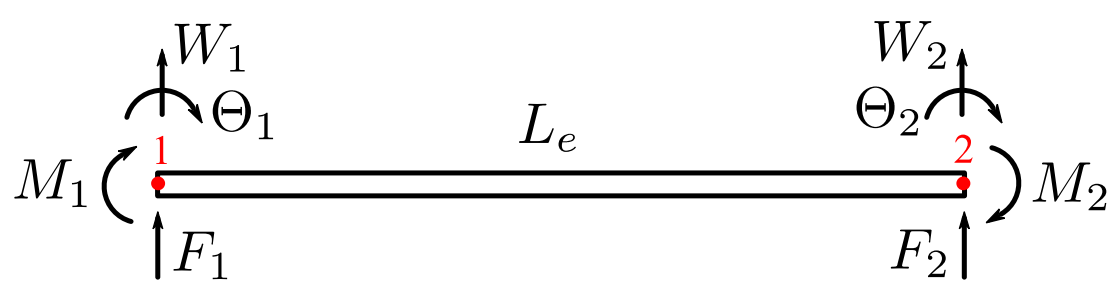

Figure 2. A beam element showing the forces, moments and linear, angular displacements at each end in local coordinate system. 
The beam element vector of forces and moments, $\mathbf{f}_{e}=\left\{\begin{array}{llll}F_{1} & M_{1} & F_{2} & M_{2}\end{array}\right\}^{T}$ is related to the vector of displacements and rotations $\quad \mathbf{w}_{e}=\left\{\begin{array}{llll}W_{1} & \Theta_{1} & W_{2} & \Theta_{2}\end{array}\right\}^{T}$ by the dynamic stiffness matrix $\mathbf{D}_{e}$, so that [46]

$$
\mathbf{f}_{e}=\mathbf{D}_{e} \mathbf{w}_{e}
$$

where

$$
\mathbf{D}_{e}=\left[\begin{array}{cccc}
-K_{11} & -P & K_{12} & V \\
-P & Q_{11} & -V & Q_{12} \\
K_{12} & -V & -K_{11} & P \\
V & Q_{12} & P & Q_{11}
\end{array}\right]
$$

in which

$$
\begin{aligned}
& K_{11}=E I \beta^{3}\left(\cos \left(\beta L_{e}\right) \sinh \left(\beta L_{e}\right)+\sin \left(\beta L_{e}\right) \cosh \left(\beta L_{e}\right)\right) / R \\
& K_{12}=E I \beta^{3}\left(\sin \left(\beta L_{e}\right)+\sinh \left(\beta L_{e}\right)\right) / R \\
& P=E I \beta^{2}\left(\sin \left(\beta L_{e}\right) \sinh \left(\beta L_{e}\right)\right) / R \\
& V=E I \beta^{2}\left(\cos \left(\beta L_{e}\right)-\cosh \left(\beta L_{e}\right)\right) / R \\
& Q_{1}=E I \beta\left(\cos \left(\beta L_{e}\right) \sinh \left(\beta L_{e}\right)-\sin \left(\beta L_{e}\right) \cosh \left(\beta L_{e}\right)\right) / R \\
& Q_{12}=E I \beta\left(\sin \left(\beta L_{e}\right)-\sinh \left(\beta L_{e}\right)\right) / R \\
& R=\cos \left(\beta L_{e}\right) \cosh \left(\beta L_{e}\right)-1
\end{aligned}
$$

and $\beta=\left(\omega^{2} \rho S / E I\right)^{(1 / 4)}$ is the flexural wavenumber, where $\omega$ is the frequency, $\rho$ is the density, $E$ is the Young's modulus, $S$ is the cross sectional area and $I$ is the second moment of area.

The model of the system shown in Fig. 1 is obtained using a procedure similar to the finite element method, however, in this approach, each beam element contains distributed mass and stiffness. The number of elements $N$ is related to the number of nodes $N+1$, which is determined by the number of points along the beam where a suspended mass, excitation force and response output is located. Each suspended mass is connected to a node between two beam elements through a parallel combination of a spring and a damper. The global dynamic stiffness matrix $\mathbf{D}$ is given by 


$$
\mathbf{D}=\sum_{e=1}^{N} \mathbf{B}_{e}^{T} \mathbf{D}_{e} \mathbf{B}_{e}+\sum_{i=1}^{N_{m}} \mathbf{C}_{i}^{T} D_{m} \mathbf{C}_{i}
$$

where $e$ is the beam element number, $\mathbf{B}_{e}$ is a Boolean matrix which converts the local node coordinates to global coordinates and $\mathbf{D}_{e}$ is the dynamic stiffness matrix given in Eq. (1). The second summation in Eq. (2) refers to the contribution of each suspended mass. The total number of suspended masses is $N_{m}$ and $C_{i}$ is also a Boolean matrix which has the function of adding $D_{m}$ at the appropriate global coordinate. The dynamic stiffness for each suspended mass is $D_{m}=-\omega^{2} m(k+\mathrm{j} \omega c) /\left(k-\omega^{2} m+\mathrm{j} \omega c\right)$ [46], where $m$ is the mass, $k$ is the stiffness, $c$ is the damping and $\mathrm{j}=\sqrt{-1}$.

The Boolean matrix $\mathbf{B}_{e}$ has four lines and $2 n$ columns, consisting of zeros and ones. The elements in the matrix are unity when the degrees of freedom of the beam element coincide with the global degrees of freedom, and are zero otherwise. Examples of the Boolean matrices for the first two beam elements are given below as

$$
\mathbf{B}_{1}=\left[\begin{array}{llllllll}
1 & 0 & 0 & 0 & 0 & \cdots & 0 & 0 \\
0 & 1 & 0 & 0 & 0 & \cdots & 0 & 0 \\
0 & 0 & 1 & 0 & 0 & \cdots & 0 & 0 \\
0 & 0 & 0 & 1 & 0 & \cdots & 0 & 0
\end{array}\right]^{4 \times 2 n}, \mathbf{B}_{2}=\left[\begin{array}{lllllllll}
0 & 0 & 1 & 0 & 0 & 0 & 0 & \cdots & 0 \\
0 & 0 & 0 & 1 & 0 & 0 & 0 & \cdots & 0 \\
0 & 0 & 0 & 0 & 1 & 0 & 0 & \cdots & 0 \\
0 & 0 & 0 & 0 & 0 & 1 & 0 & \cdots & 0
\end{array}\right]^{4 \times 2 n}
$$

Similarly, the matrix $\mathbf{C}_{i}$ has one row and $2 n$ columns. The elements of $\mathbf{C}_{i}$ are zeros, except for the degree of freedom where the suspended mass $i$ is attached. For example, the Boolean matrix for the last suspended mass is given by

$$
\mathbf{C}_{N_{m}}=\left[\begin{array}{lllll}
0 & 0 & \cdots & 1 & 0
\end{array}\right]^{1 \times 2 n}
$$

After applying the boundary conditions, which correspond to fixed-free conditions for the system in Fig. 1, the velocity at each node is given by

$$
\mathbf{v}=\mathrm{j} \omega \mathbf{D}^{-1} \mathbf{f}
$$


where $\mathbf{v}$ is the global vector of linear and angular velocities and $\mathbf{f}$ is the global vector of forces and moments, which has zero elements, except at the degree of freedom related to the applied external force.

Numerical simulations are used to illustrate the behaviour of the system in Fig. 1 with the parameters given in Table 1. In the first simulation, three masses are located at distances 0.47 $\mathrm{m}, 0.73 \mathrm{~m}$ and $1 \mathrm{~m}$, respectively, from the fixed end of the 1-meter-length beam (the locations were selected so that they were not at nodal points in the frequency range of interest). Note that the stiffness that connects each mass to the beam is very small, so that the natural frequency of each suspended mass arrangement is well below all natural frequencies of the beam.

Table 1. Physical properties used in the numerical simulations

\begin{tabular}{ll}
\hline Property & Value \\
\hline Beam Young's Modulus, $E$ & $210 \times 10^{9} \mathrm{~N} / \mathrm{m}^{2}$ \\
Beam density, $\rho$ & $7800 \mathrm{~kg} / \mathrm{m}^{3}$ \\
Beam length $\times$ width $\times$ thickness & $1 \times 0.2 \times 0.012 \mathrm{~m}$ \\
Each suspended mass, $m$ & $5 \%$ of beam mass \\
Each viscous damping element, $c$ & $250 \mathrm{Ns} / \mathrm{m}$ \\
Each quasi-zero stiffness, $k$ & $1 \mathrm{~N} / \mathrm{m}$ \\
Location of the force from the fixed end & $0.2 \mathrm{~m}$ \\
Beam loss factor & 0.01 \\
\hline
\end{tabular}

The transfer mobility of the velocity of the free end of the beam per unit input force is shown in Fig. 3, along with a similar simulation is with a single suspended mass located at the freeend of the beam. It can be seen from Figure 3 that the effects of adding three suspended masses or one single mass are qualitatively similar. The suspended masses cause two main effects - an increase in the overall damping of the structure, and a small downward shift of the lowest resonance frequencies. The damping effect on each mode is dependent on the position of the masses relative to the nodal points. Since the influence of either one or three masses is very similar, the subsequent discussion is limited to the case of a beam with a single suspended mass only. 


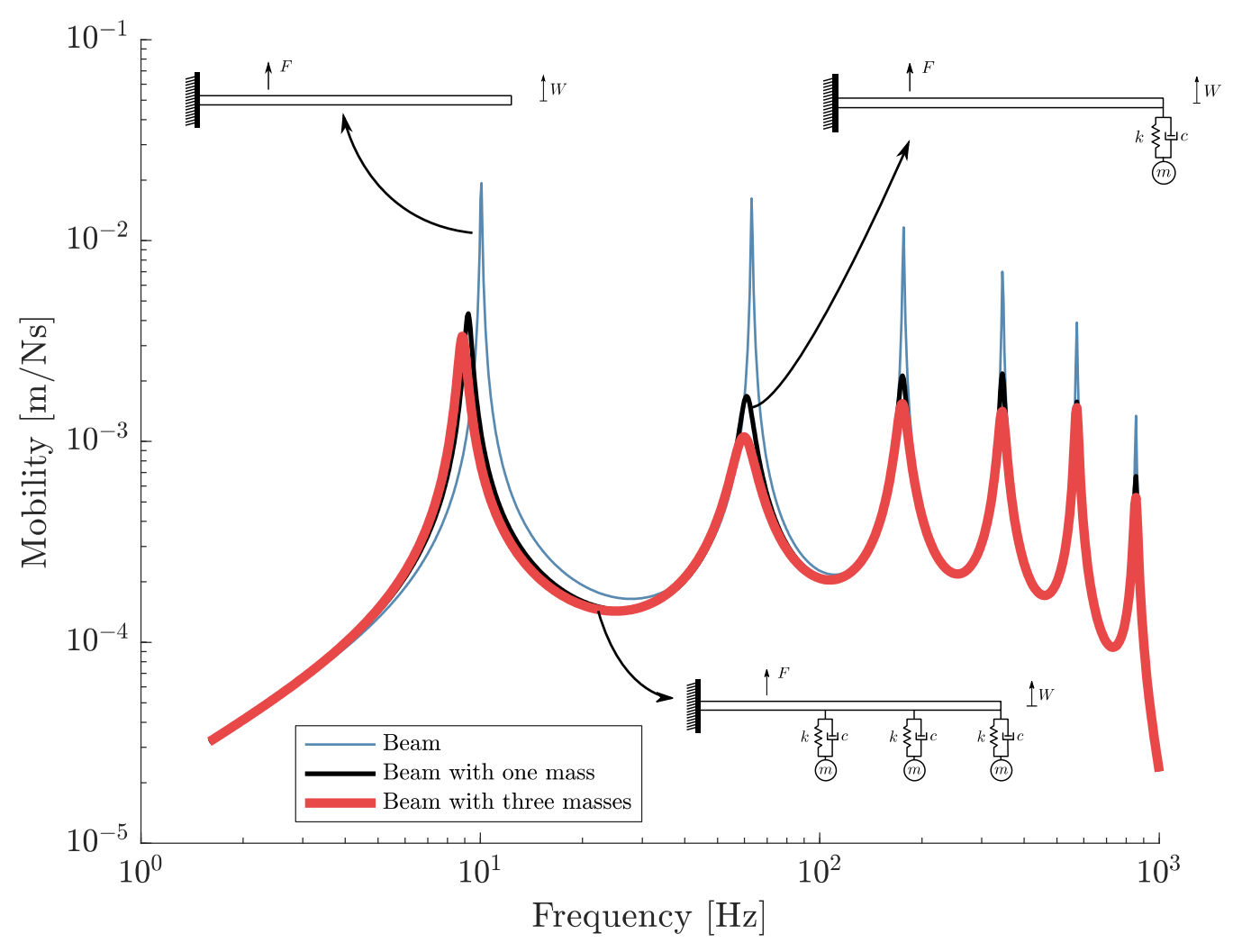

Figure 3. Comparison of the mobility response of the beam with three suspended masses, and one suspended mass. Also plotted is the mobility of the beam alone.

To help in the interpretation of the results in Fig. 3, the $D_{j}$ term in Eq. (2) is examined in more detail. As the suspension stiffness is very small, in the frequency range of interest is $\omega>>\sqrt{k / m}$, so that $D_{j} \approx-\mathrm{j} \omega^{2} m c /(-\omega m+\mathrm{j} c)$. In the case when $\omega<<c / m$, then $D_{n} \approx-\omega^{2} m$, i.e., there is mass-like behaviour; and when $\omega>>c / m$, then $D_{n} \approx-\mathrm{j} \omega c$, i.e., there is damping-like behaviour. Thus, the four cases illustrated in Figs. 4(a)-(d) are considered. The simulations are presented in Fig. 5. For clarity, cases (a), (b) and (c) are shown separately in Fig. 5(a), and cases (a), (b) and (d) are shown in Fig. 5(b). 
(a)

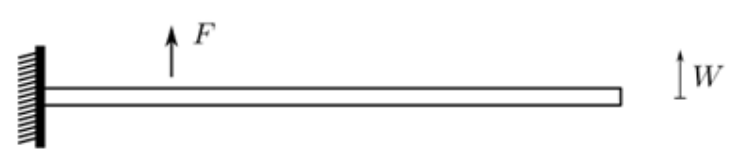

(c)

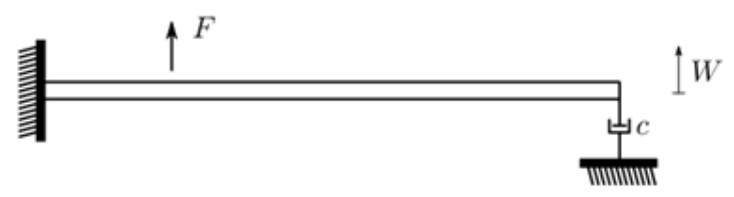

(b)

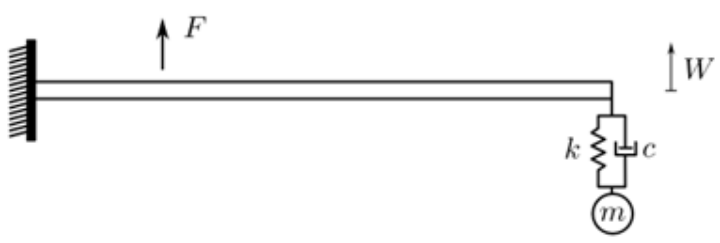

(d)

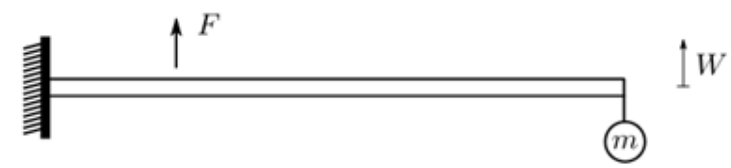

Figure 4. Different configurations of a cantilever beam subject to harmonic force. (a) Beam alone, (b) beam with mass attached through viscous damper and quasi-zero stiffness, (c) beam with grounded viscous damper attached (d) beam with mass attached.

In Fig. 5(a), it can be seen that at lower frequencies, for example close to the first resonance frequency, the response of the beam with suspended mass is different to the case of the beam with grounded damper. However, when the frequency is increased, that difference becomes less and less significant. In Fig. 5(b) it can be seen that at relatively low frequencies, the response of the beam with suspended mass is very similar to the case of the beam with directly attached mass. Thus, if the natural frequency of the structure is much less (greater) than $c / m$ then the attachment will predominantly act like an added mass (damper). An animation that highlights these effects is supplied in supplementary material for this paper. 
(a)

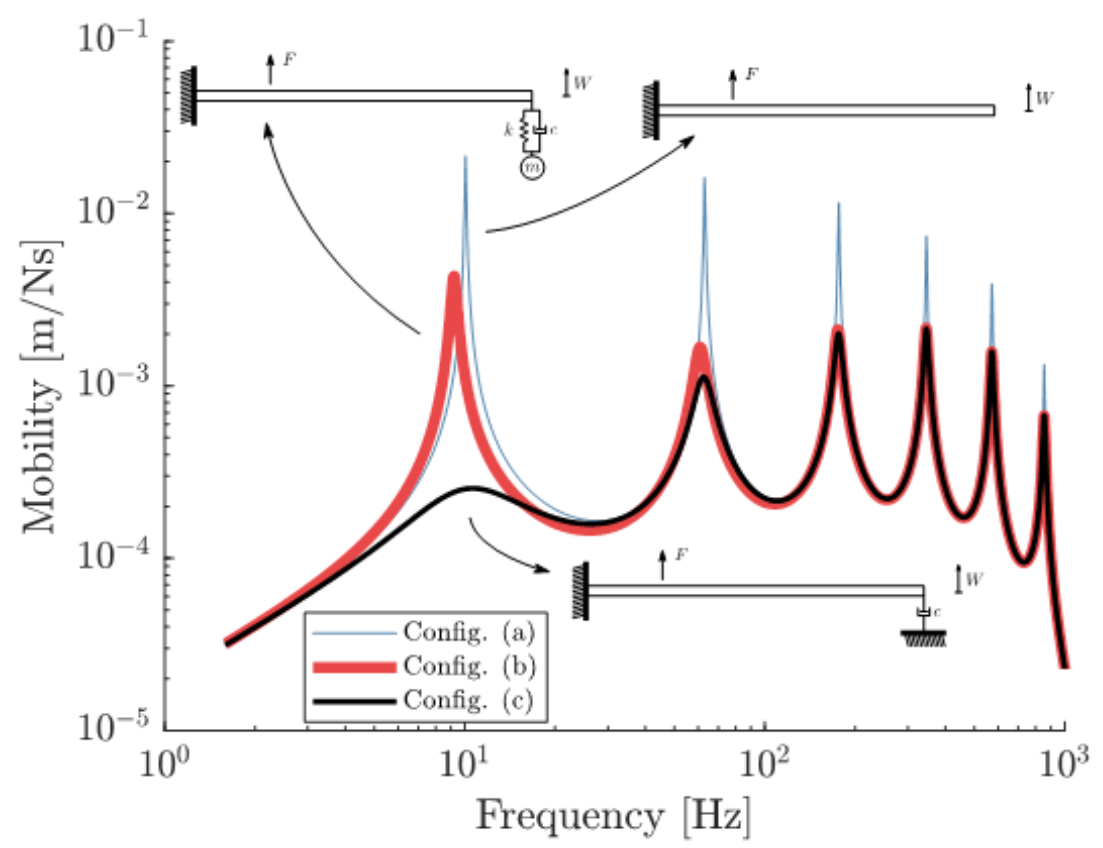

(b)

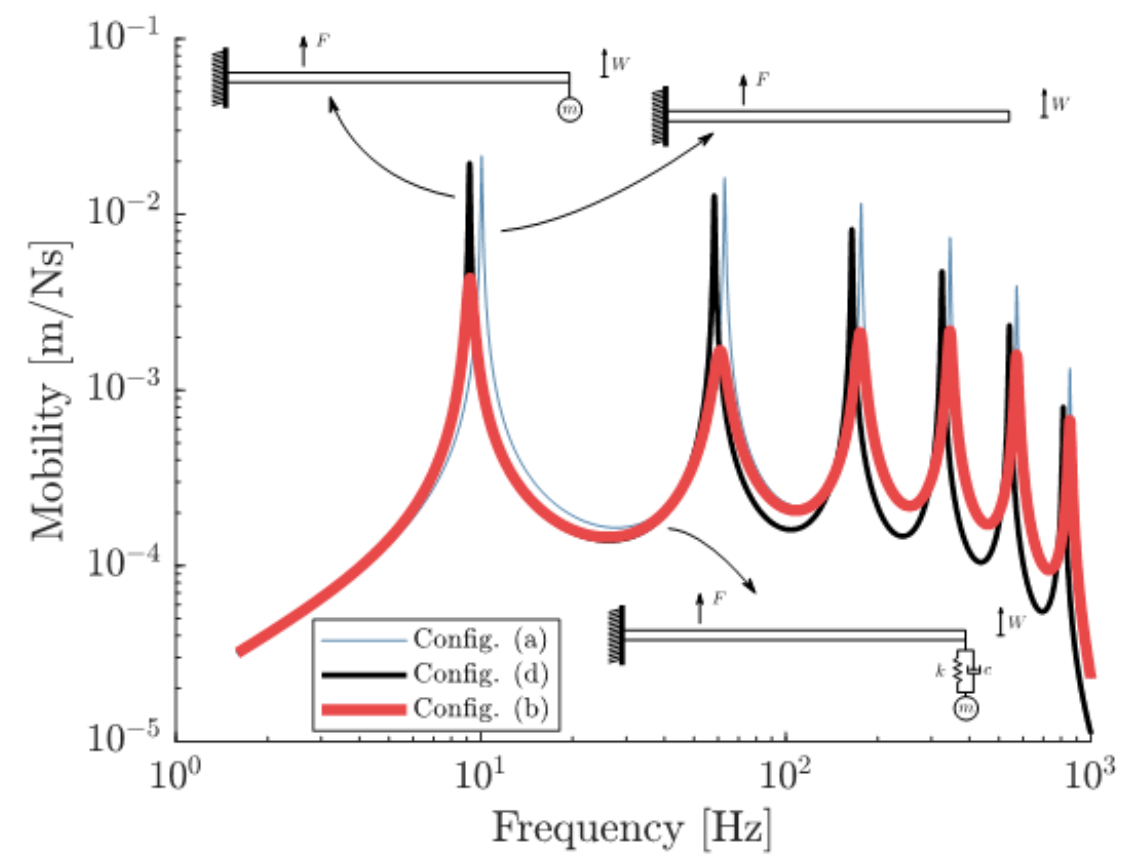

Figure 5. Comparison of mobility responses. (a) Beam without and with low-stiffness suspended mass compared to the beam with viscous damper. (b) Beam without and with low-stiffness suspended mass compared to the beam with directly attached mass. 


\section{THEORETICAL DESIGN OF A NONLINEAR STIFFNESS ELEMENT FOR QZS}

This section presents a mathematical description of a QZS device which has two simple adjustments - one to correct stiffness errors, and a second to correct for errors in static loading. Like many nonlinear devices (e.g. $[44,45])$ the suspension system consists of two parts or subsystems. The first of these supports the static load, and is shown in Fig. 6(a).
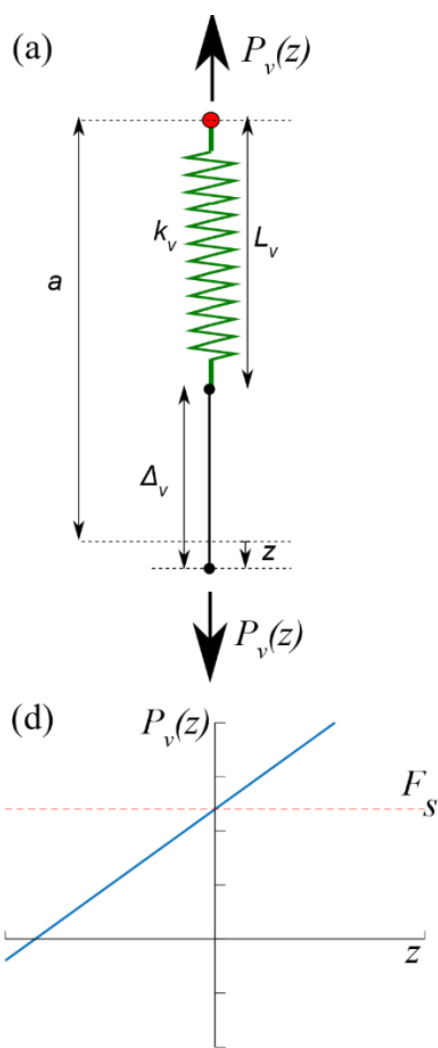

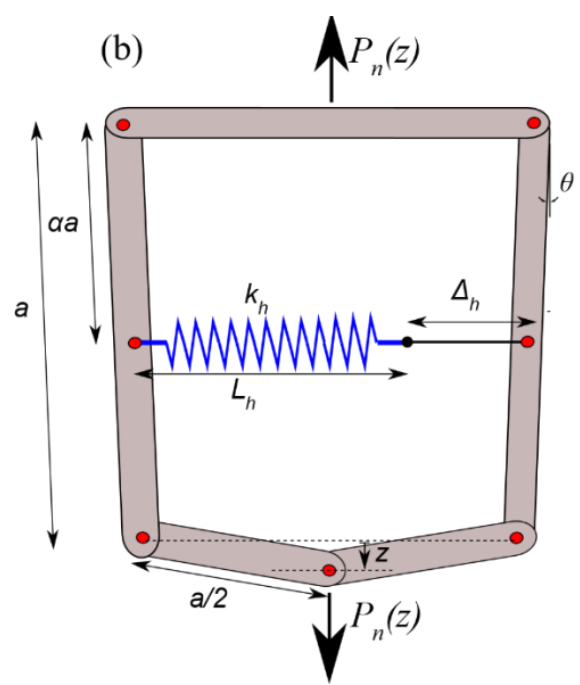

(e)

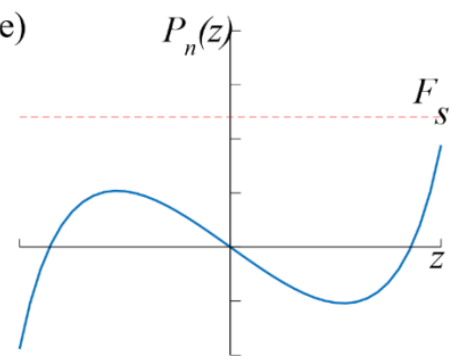

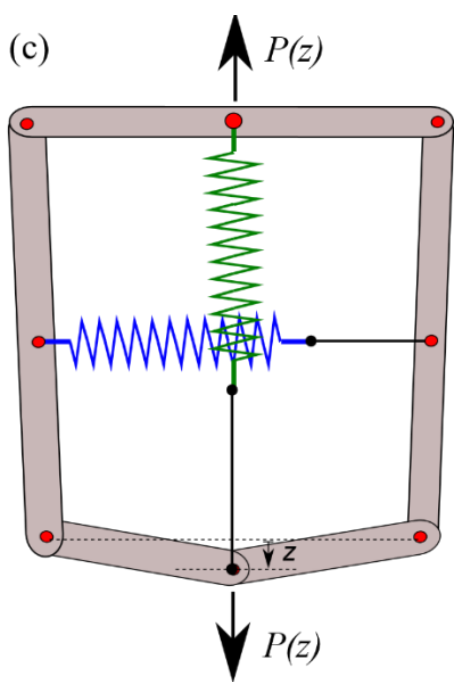

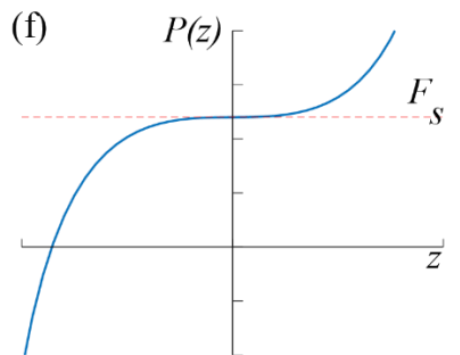

Figure 6. Subsystems of the QZS suspension and associated force displacement curves, with associated mathematical symbols. (a) Vertical spring component, providing positive stiffness and static load support. (b) Negative stiffness mechanism (note horizontal spring remains under tension at all times during normal operation). (c) Combination of (a) and (b) to give the QZS characteristic. (d-f) Typical force displacement responses for parts (a-c), respectively. 
In this implementation, as in many others (e.g. $[40,44])$, this part simply consists of a linear spring oriented vertically. With reference to the parameters labelled in Fig. 6, the forcedisplacement relationship for the vertical spring of stiffness $k_{v}$, is given by

$$
P_{v}(z)=k_{v}\left(l_{v}-l_{0 v}\right)+F_{0 v}=k_{v}\left(a+z-\Delta_{v}-L_{0 v}\right)+F_{0 v}
$$

where $L_{0 v}$ is the initial length of the spring and $F_{0 v}$ is the tension required to start extending the spring from this position. Note that by varying $\Delta_{v}$ the spring can be adjusted so that the static load is supported at the chosen initial position, i.e., $P_{v}(0)=F_{s}$ for different values of the static load $F_{s}$.

The second subsystem is shown in Fig. 6(b) and adds the required negative stiffness and resulting nonlinearity. Note that at $z=0$ the linkage forms a square of side $a$. The elastic potential energy within this system is solely due to the horizontal spring of stiffness $k_{h}$ and is given by

$$
V_{h}=\frac{k_{h}}{2}\left(L_{h}-L_{0 h}\right)^{2}+F_{0 h}\left(L_{h}-L_{0 h}\right)
$$

where $L_{0 h}$ is the initial length of the spring and $F_{0 h}$ is the tension required to start extending the spring from this position. Note from Fig. 6(b) that the distance $L_{h}+\Delta_{h}$ is given by

$$
L_{h}+\Delta_{h}=a-\alpha\left(a-2 \sqrt{\left(\frac{a}{2}\right)^{2}-z^{2}}\right)
$$

so that

$$
q(z)=L_{h}-L_{0 h}=a-\alpha\left(a-2 \sqrt{\left(\frac{a}{2}\right)^{2}-z^{2}}\right)-\Delta_{h}-L_{0 h} .
$$

Combining Eqs. (5) and (7) and differentiating the potential energy, results in the vertical force due to the horizontal spring, given by

$$
P_{n}(z)=\frac{\mathrm{d} V_{h}}{\mathrm{~d} q} \frac{\mathrm{d} q}{\mathrm{~d} z}=\left(k_{h} q(z)+F_{0 h}\right) \frac{-2 \alpha z}{\sqrt{\left(\frac{a}{2}\right)^{2}-z^{2}}}
$$


Note that the effect on the displacement $z$ of the rotation of the vertical members in Fig. 6(b) is neglected. Figure 6(e) shows a typical shape of the force displacement function described by Eq. (8). The stiffness at $z=0$ is found from the derivative of the force-deflection curve, and is given by

$$
\left.\frac{\mathrm{d} P_{n}}{\mathrm{~d} z}\right|_{z=0}=\frac{-4 \alpha}{a}\left(k_{h}\left[a-\Delta_{h}-L_{0 h}\right]+F_{0 h}\right),
$$

in which it can be seen that the negative stiffness of this subsystem can be varied by adjusting $\Delta_{h}$. The parallel combination of these two subsystems is shown in Fig. 6(c), and the forcedisplacement characteristic is determined by summing the forces given in Eqs. (4) and (8) to give

$$
P(z)=P_{v}(z)+P_{n}(z)
$$

A typical force-deflection curve for the complete device is shown in Fig. 6(e). Note from Eq. (8) that $P_{n}(0)=0$, so that

$$
P(0)=P_{v}(0)=k_{v}\left(a-\Delta_{v}-L_{0 v}\right)+F_{v 0} .
$$

To achieve the requirement that $P(0)=F_{s}, \Delta_{v}$ is adjusted so that

$$
\Delta_{v}=\frac{F_{0 v}-F_{s}}{k_{v}}+a-L_{0 v}
$$

The overall stiffness of the device at $z=0$ is given by

$$
\left.\frac{\mathrm{d} P}{\mathrm{~d} z}\right|_{z=0}=\left.\frac{\mathrm{d} P_{n}}{\mathrm{~d} z}\right|_{z=0}+\left.\frac{\mathrm{d} P_{v}}{\mathrm{~d} z}\right|_{z=0}=\frac{-4 \alpha}{a}\left(k_{h}\left[a-\Delta_{h}-L_{0 h}\right]+F_{0 h}\right)+k_{v},
$$

and this gives the overall stiffness of the device $k_{e}$ if equilibrium is achieved at $z=0$. Hence any required $k_{e}$ can be found by choosing

$$
\Delta_{h}=\frac{a\left(k_{e}-k_{v}\right)}{4 \alpha}+a-L_{0 h}+\frac{F_{0 h}}{k_{h}} .
$$


Therefore, the QZS mechanism can, in principle, be made free from static loading error, simply by setting $\Delta_{v}$ in accordance with Eq. (12). Then any required value for $k_{e}$ can be achieved by simply choosing $\Delta_{h}$ in accordance with Eq. (14).

\section{EXPERIMENTAL TESTS ON THE DEVICE}

\subsection{Prototype of the Device}

A prototype of the QZS device is illustrated in Figs. 7(a) and (b). The distance $a$ between the joints in Fig. 6, is $70 \mathrm{~mm}$, and each arm is made from a $4 \mathrm{~mm}$ aluminium plate. Good quality roller bearings are used for the five joints.

To permit accurate adjustment of the parameters $\Delta_{h}$ and $\Delta_{v}$, the device was equipped with two tuning pegs such as those found on guitars or other stringed instruments. The tuners have an internal worm screw that is self-locking and gives a gear ratio of 18:1. The peg diameter is nominally $6 \mathrm{~mm}$ although the effective diameter will vary slightly when coils of thread lie on top of each other. Thus, one eighth of a revolution on the a peg adjusts $\Delta_{h}$ or $\Delta_{v}$ by approximately $0.13 \mathrm{~mm}$. Smaller adjustments are clearly possible, so this figure gives a very conservative estimate of the fidelity of the adjustment. The springs are connected to thin Dyneema ${ }^{\circledR}$ cord which is wrapped around the shaft of the tuning pegs and tied off.

It was found that as the cord for the vertical adjuster had several turns on the upper tuning peg shaft, there could be slight misalignment of the force applied by the vertical spring causing the device to skew. To overcome this problem the wire loop, visible in Fig. 7(b) was incorporated as a guide to ensure that the line of force for the vertical spring remained central. From separate static measurement, the stiffness of the horizontal and vertical springs were found to be $2.21 \mathrm{~N} / \mathrm{mm}$ and $0.718 \mathrm{~N} / \mathrm{mm}$, respectively. 
(a)

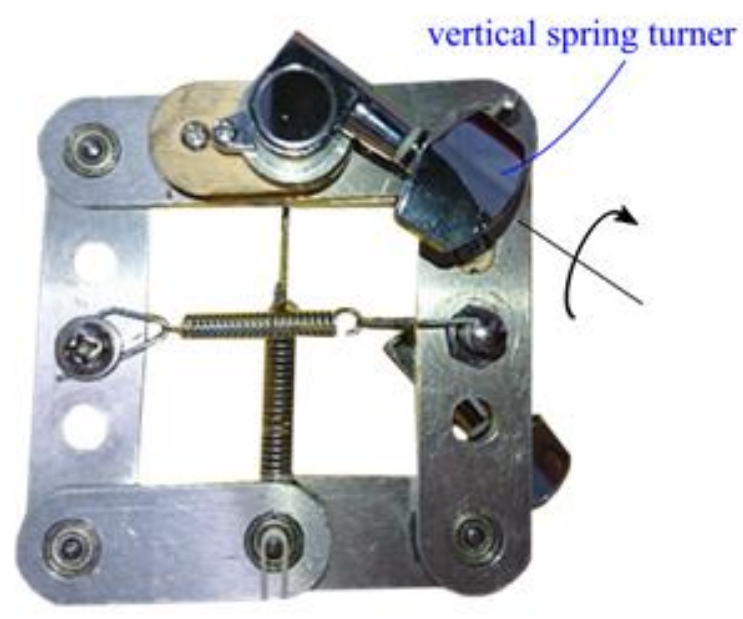

(b)

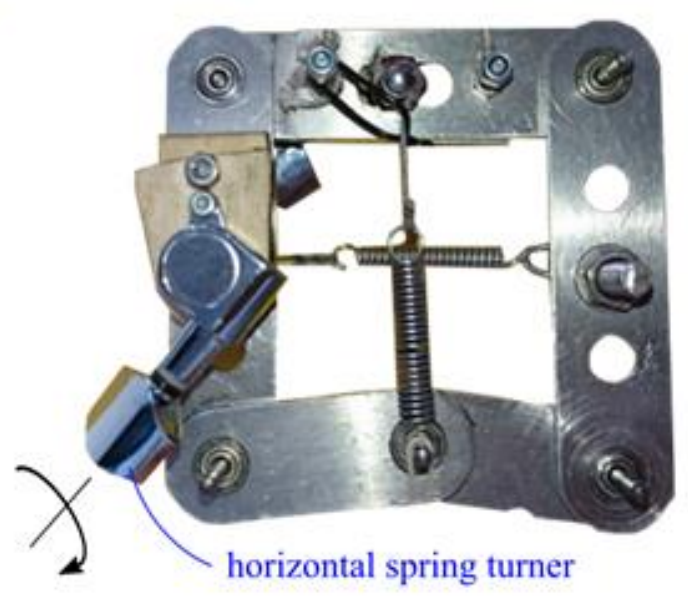

Figure 7. Photographs of the prototype QZS device showing the front (a) and the rear (b) view.

\subsection{Quasi-static characterization}

To obtain a quasi-static characterisation, the device was mounted in a ZwickiLine TH $2.5 \mathrm{kN}$ load testing machine, as shown in Fig. 8(a). The process to obtain force-displacement data was as follows:

1. The QZS device was suspended from the upper jaws of the tester (where the load cell is located) and the loading force was zeroed, hence the zero excludes the self-weight of the device.

2. The device was connected to the lower jaws of the tester, and then the upper jaws were raised until the two lower beams were horizontal. This ensured that the load test would commence from the ideal equilibrium point for the device, as currently configured.

3. An expanding saw tooth displacement cycle as shown in Fig. 8(b) was applied with a loading rate of $100 \mathrm{~mm} / \mathrm{min}$. This was used so that the repeatability of the measured forces and the effect of any hysteresis in the loading cycles could be monitored. 
(a)

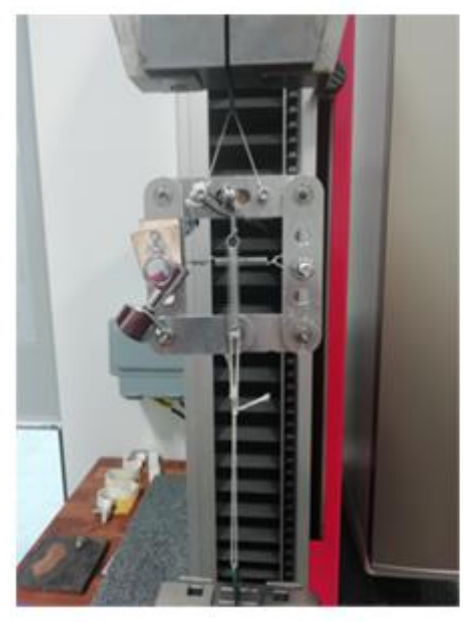

(b)

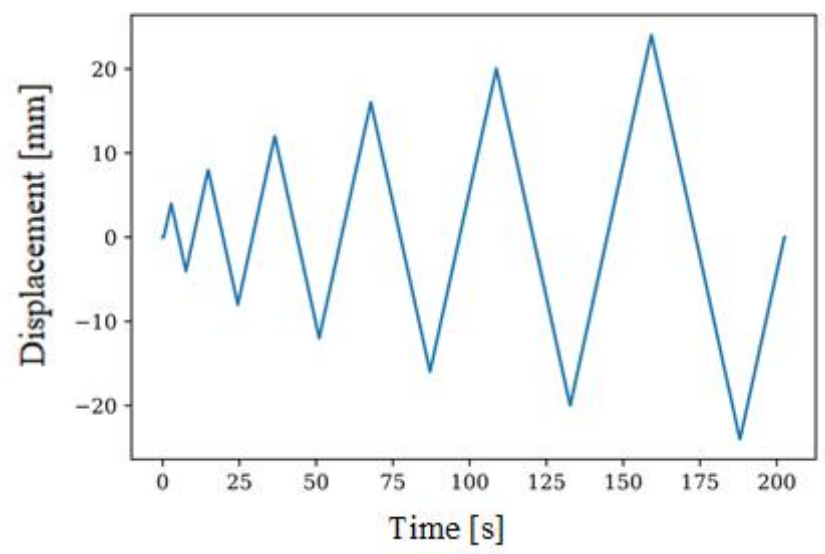

Figure 8. (a) The QZS suspension device at $z=0$ mounted in the load tester. (b) Applied displacement cycle.

A typical measured force-displacement curve as a result of this process is shown in Fig. 9. The results confirm that the mechanism has a smooth nonlinear force-displacement characteristic. Also shown are fitted $3^{\text {rd }}$ and $5^{\text {th }}$ order polynomials to the central part of the data. It can be seen that the data can be well matched by a third order polynomial. However, it is also clear that friction is significant in the device, causing hysteresis on each loading cycle. 


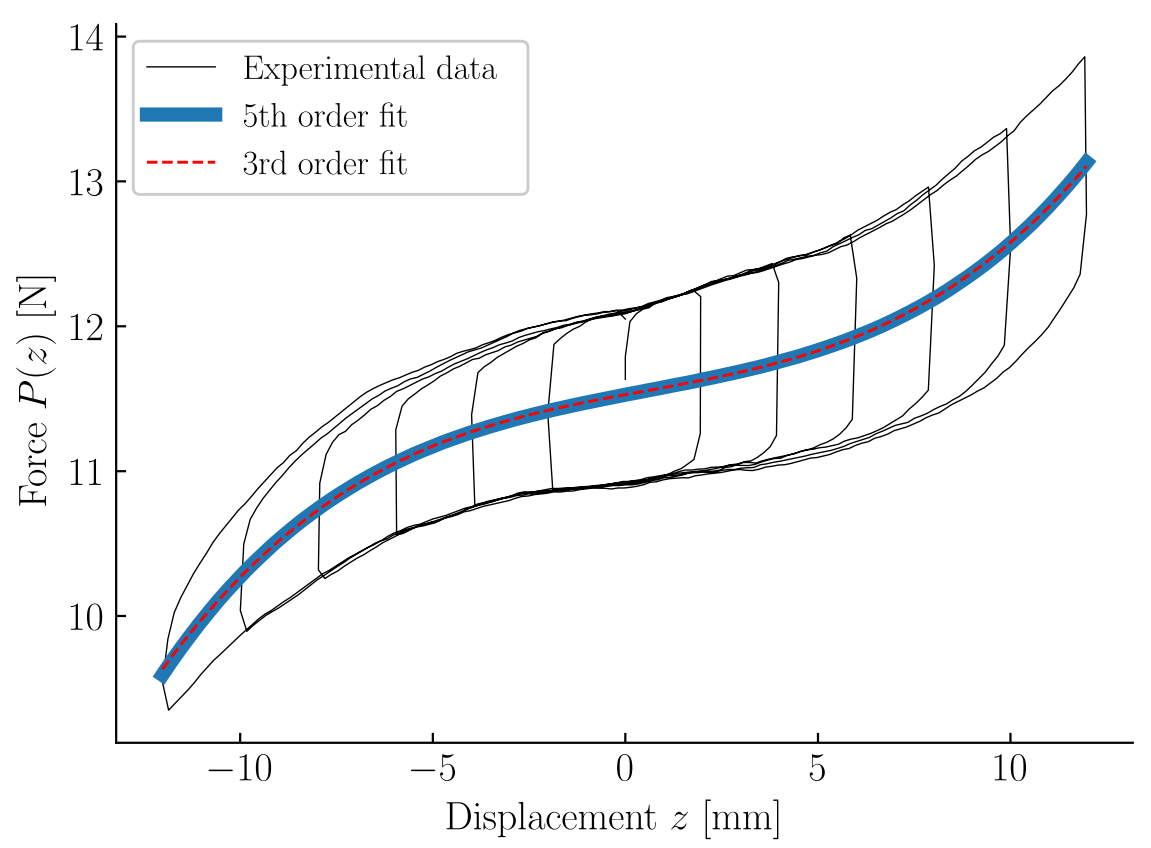

Figure 9. Results of the quasi-static tests.

Figure 10(a) shows the effect of adjustments to the vertical spring tuning peg. It can be seen that the shape of the force displacement curve remains mostly constant with this adjustment. If positive turns are applied (which increase the tension in the vertical spring) the graph is translated upwards, which may be necessary for a larger static load. Similarly, negative turns reduce the tension in the vertical spring, and this may be necessary for smaller static loads. With reference to the discussion in Section 3, Figure 10(a) shows that the vertical tuning peg effectively alters $\Delta_{v}$ and has the desired effect of adjusting for the static load, as shown in Eq. (11).

Figure 10(b) shows the effect of adjusting the horizontal spring tuning peg. Compared to the 'neutral' case, increasing the turns (and therefore increasing the horizontal spring tension, or decreasing $\Delta_{h}$ ) reduces the stiffness near zero, or even to negative values if there is an excessive number of turns. Similarly, reducing the turns on the horizontal spring tuner increases the stiffness. However, there is very little change in the static force $P(0)$ showing that adjustments to the static stiffness are effectively independent from adjustments to the static load. This tuner is effectively adjusting $\Delta_{h}$, confirming predictions made in Section 3. 
In particular this parameter has a direct effect on the zero displacement stiffness as shown by Eq. (13), whilst having no effect on the zero displacement force as predicted by Eq. (11).

Figures 10(a) and (b) therefore confirm experimentally that the device is able to effectively decouple two different effects: the vertical tuner acts on $P(0)$ only, without affecting the stiffness at $z=0$; the horizontal tuner acts on the stiffness at $z=0$ only, without affecting $P(0)$. In this way, it is possible to achieve a true QZS effect acting on the horizontal tuner only, and balancing any static load thorough the vertical tuner independently.

(a)

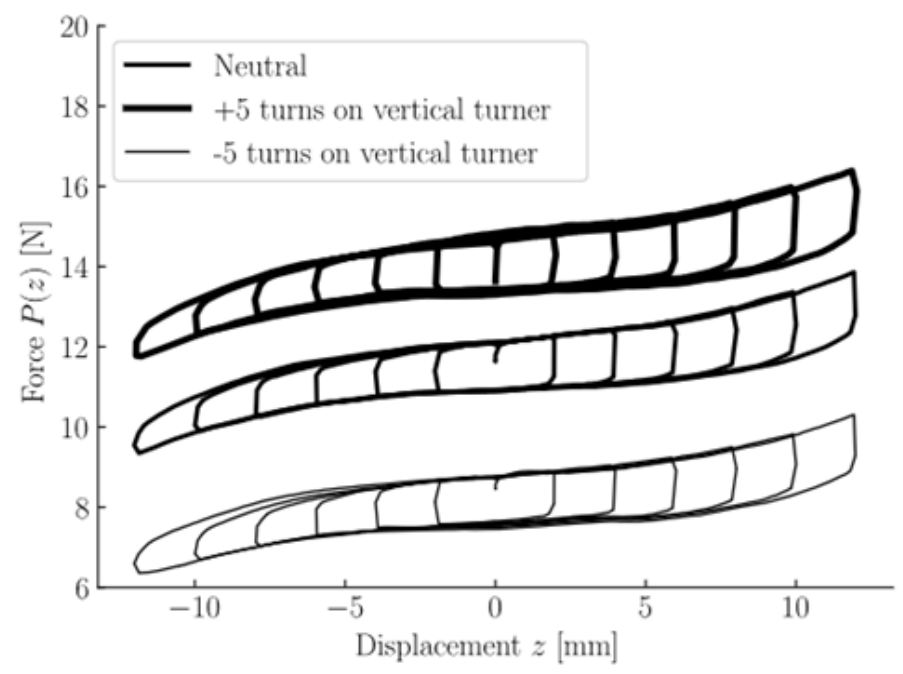

(b)

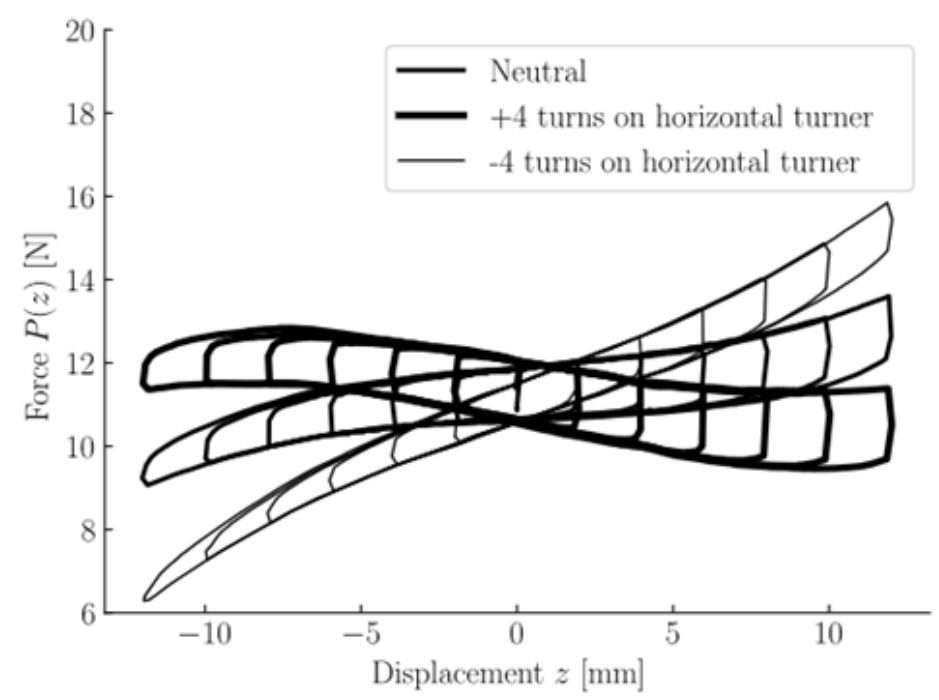


Figure 10. Results of quasi-static tests showing the effects of adjusting the tuners. (a) Adjustment of the vertical spring tuner, (b) adjustment the horizontal spring tuner. 'Neutral' denotes an arbitrary initial configuration to which the adjustments are applied.

\subsection{Dynamic characterization}

To gain insight into the dynamic performance of the QZS device, it is connected to an APS113 ELECTRO-SEIS® Long-Stroke vibration shaker at one end, and a $1.5 \mathrm{~kg}$ mass is suspended from the other end, as shown in Fig. 11. The shaker was driven by a voltage controlled sinusoidal signal, with the shaker amplifier setting at approximately half of the maximum gain. Stepped sine tests were used to capture the low frequency resonant behaviour of the system, where the displacement of both the payload mass and the shaker armature were measured by Omron laser displacement sensors.

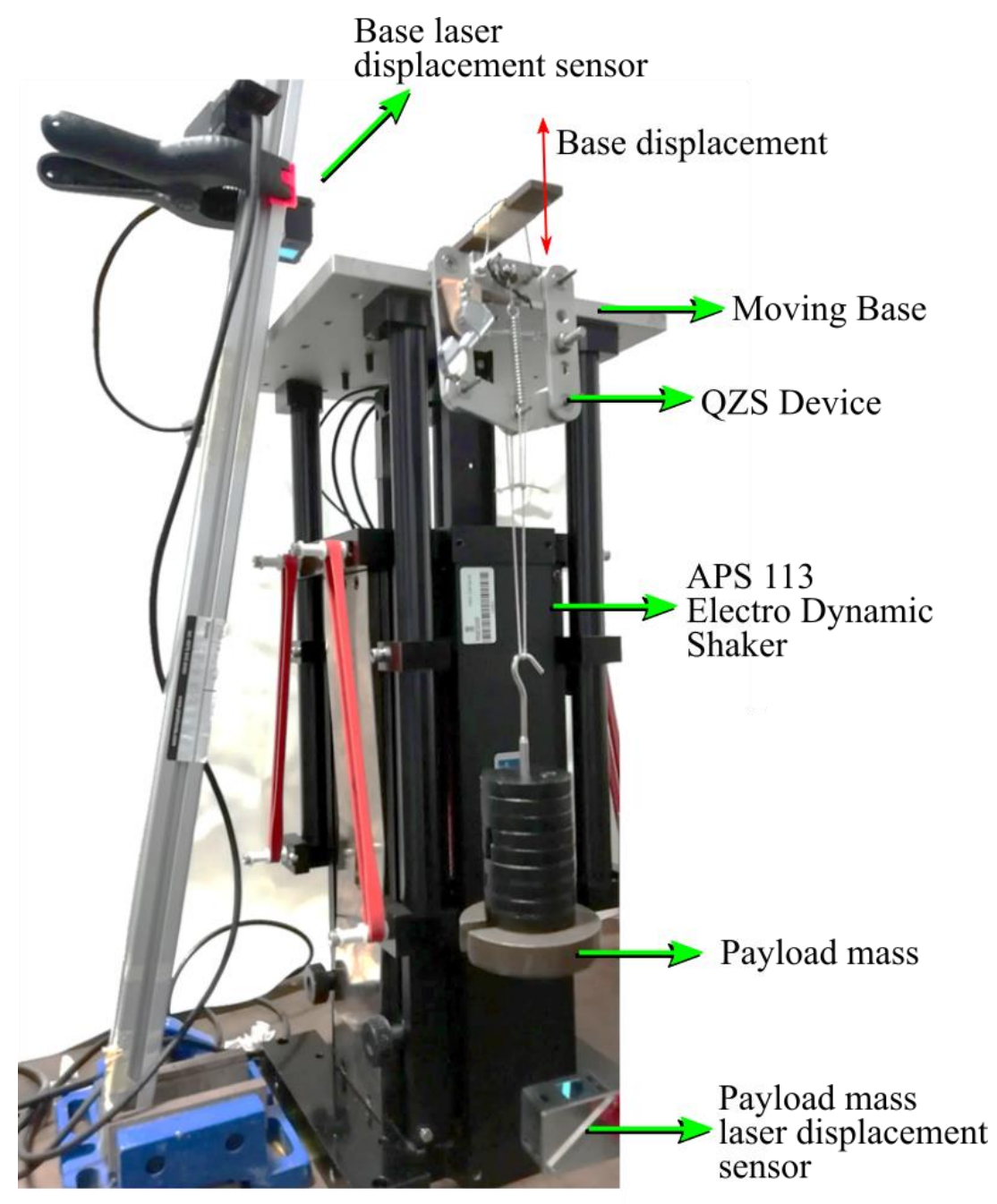

Figure 11. The QZS mechanism suspended from the APS113 shaker. 
An in-house algorithm was developed to control the stepped sine testing procedure, with a settling criterion used at each data point, to strike a balance between the quality of data and achieving reasonably short sweep times. The settling algorithm involved the repeated sampling of periods of data, forming a vector of the first 10 Fourier components (sine and cosine components of the first 5 harmonics) of the base displacement signal. After a minimum of 5 forcing periods, if the relative difference between two successive vectors was less than $10 \%$, the result was accepted. However, to limit the time over which a test could take, a maximum of 30 settling periods was permitted, after which a data point was taken anyway.

An initial stepped sine test was conducted with the horizontal tuner adjusted until it felt by hand to be near zero stiffness. The vertical spring tuner was then adjusted until the lower arms of the mechanism lay horizontally under the weight of the payload. The sweep initially progressed upwards then downwards for frequencies between $0.5 \mathrm{~Hz}$ and approximately 100 $\mathrm{Hz}$, at logarithmic intervals. Data up to $5 \mathrm{~Hz}$ is presented in Fig. 12, because higher frequency data became quite noisy as the response of the payload mass was very small due to the isolating effect of the QZS device. 
(a)

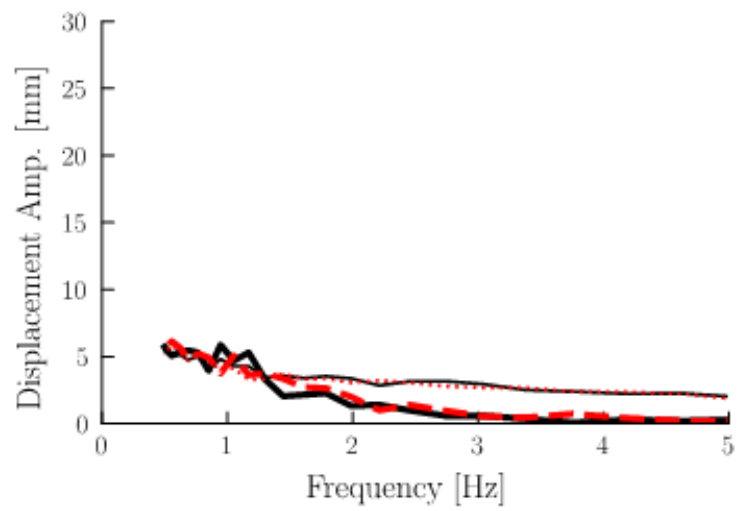

(c)

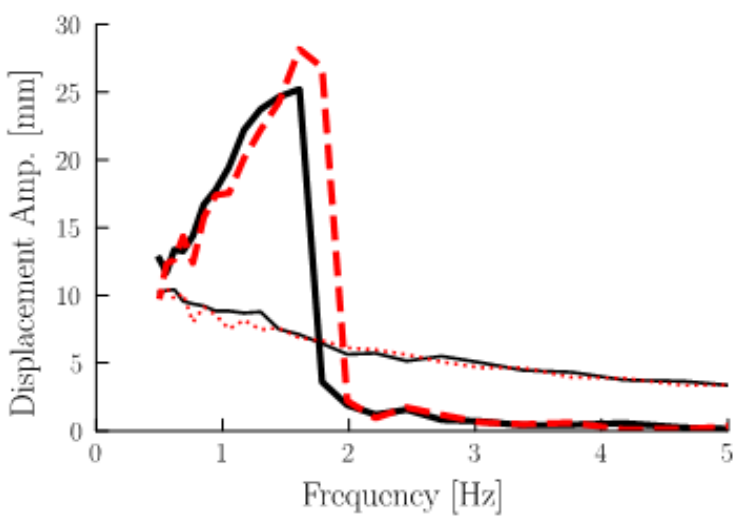

(b)

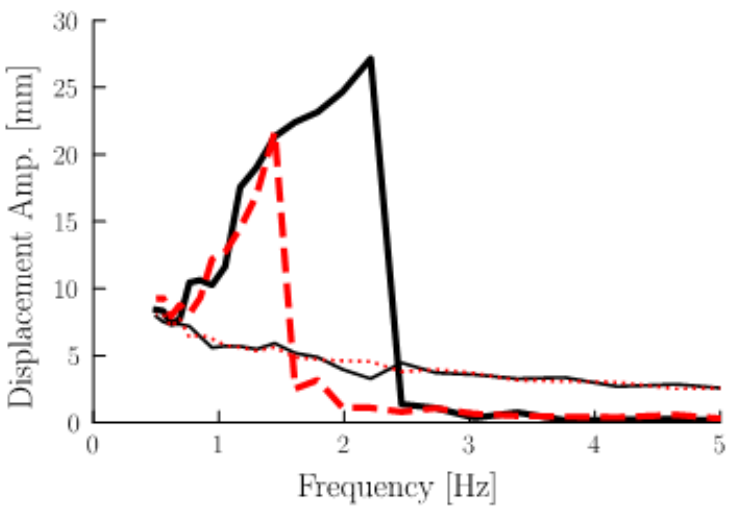

Figure 12. Absolute displacement response amplitudes for initial configuration, with voltage amplitudes (a) $0.4 \mathrm{~V}$, (b) $0.5 \mathrm{~V}$, (c) $0.6 \mathrm{~V}$.

Examination of Fig. 12(a) shows that at very low frequencies, the friction within the mechanism is sufficient to completely suppress the response at the resonance frequency. In Fig. 12(b) and (c) a classic hardening response can be seen for relatively high amplitudes of oscillation - the frequency response curve bends to the higher frequencies so that jump-up and jump-down can occur [48]. This is particularly evident in Fig. 12(b), where the sweep up causes a sudden jump-down at about $2.5 \mathrm{~Hz}$, while the sweep down causes a sudden jump-up at a lower frequency, about $1.8 \mathrm{~Hz}$. Note, however, that during the upsweep part of the test for a $0.6 \mathrm{~V}$ supply shown in Fig. 12(c) the motion became excessively large at approximately $1.8 \mathrm{~Hz}$, and needed some manual restraint to prevent damage. Thus, caution is necessary when interpreting the data around this frequency. 
Following the initial dynamic test, the QZS device was transferred back to the Zwick load tester, and a quasi-static measurement as described in Section 4.1 (with a greater range of displacement) was performed. The results of this test are shown in Fig. 13(a), where it can be seen that the suspension has a very low equilibrium stiffness.

For comparison, a model of form

$$
P(z)=p_{0}+p_{1} z+p_{2} z^{2}+p_{3} z^{3}+F_{c} \operatorname{sgn}(\Delta z) .
$$

is overlaid, where the constants $p_{i}$ are the polynomial stiffness coefficients, $F_{c}$ is force due to Coulomb friction, and $\operatorname{sgn}(\Delta z)$ is equal to 1 when the loader is advancing in the increasing $z$ direction and -1 otherwise. The unknown coefficients are fitted using linear regression and their values are given in Table 2.

Table 2 Fitted coefficients for Fig. 13.

\begin{tabular}{clll}
\hline \multirow{2}{*}{ Property } & \multirow{3}{*}{ Units } & \multicolumn{2}{l}{ Value } \\
\cline { 3 - 4 } & & (a) & $(\mathrm{b})$ \\
\hline$p_{0}$ & $\mathrm{~N}$ & 1.492 & 1.487 \\
$p_{1}$ & $\mathrm{~N} / \mathrm{mm}$ & $1.314 \times 10^{-2}$ & $-1.822 \times 10^{-2}$ \\
$p_{2}$ & $\mathrm{~N} / \mathrm{mm}^{2}$ & $-9.480 \times 10^{-4}$ & $3.497 \times 10^{-4}$ \\
$p_{3}$ & $\mathrm{~N} / \mathrm{mm}^{3}$ & $6.686 \times 10^{-4}$ & $6.738 \times 10^{-4}$ \\
$F_{c}$ & $\mathrm{~N}$ & $3.623 \times 10^{-1}$ & $4.034 \times 10^{-1}$ \\
\hline
\end{tabular}


(a)

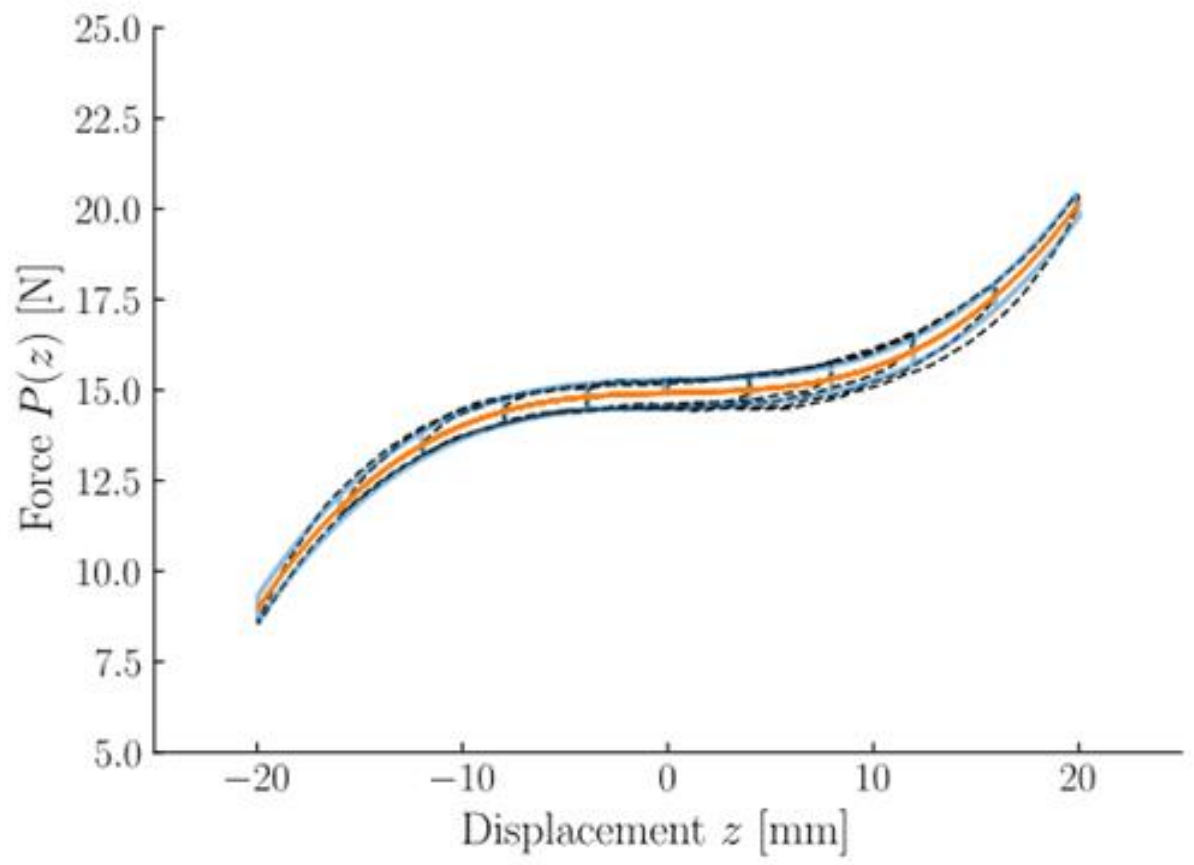

(b)

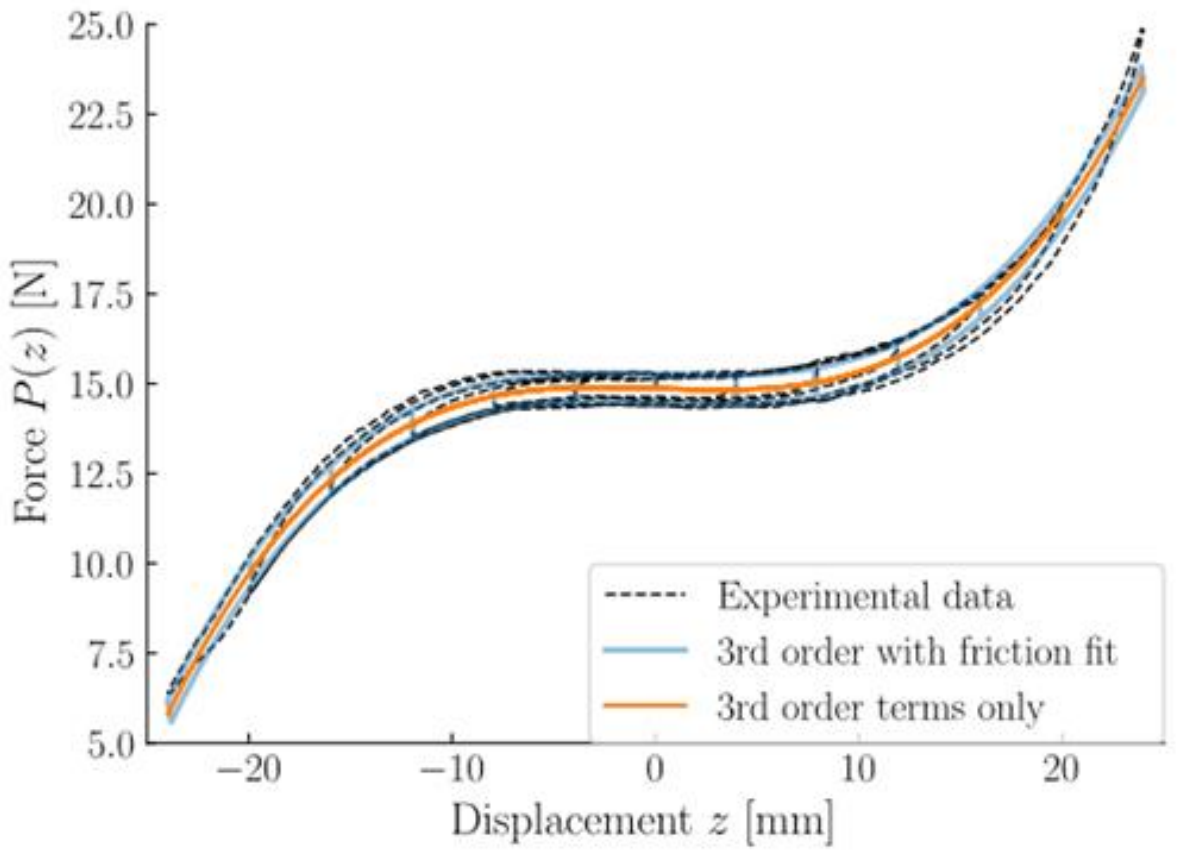

Figure 13. Quasi-static force displacement data for (a) initial configuration and (b) refined configuration. 
To determine if any further reduction in the resonant frequency of the mass-QZS system could be achieved, the horizontal stiffness was altered using the horizontal adjuster, while repeated quasi-static measurements were made. The final result shown in Fig. 13(b) was visually judged to be the closest to zero equilibrium stiffness - note that this is somewhat ambiguous as the upper curves of the hysteresis loop show a slightly positive trend, while the lower curves show a slightly negative trend around the static equilibrium position. A cubic polynomial that was subsequently fitted to that data, suggests a slightly negative equilibrium stiffness. However, no bi-stable behaviour was discernible, which is perhaps because the negative stiffness region lies entirely within the region where friction could suppress this. In this sense, friction performs a positive role in stabilizing a very low or even negative stiffness system.

Figure 14 shows the dynamic performance of the refined configuration, when the QZS device was placed in the test rig shown in Fig. 11. In this case, it can be seen that the resonance almost completely disappears at an excitation amplitude of $0.5 \mathrm{~V}$, suggesting that the resonant peak has been driven to even lower frequencies where friction locking suppresses the dynamic response. Figures 14 and 15 highlight an interesting feature of the true performance of QZS isolators, which is that it is almost impossible to observe the near zero frequency resonance, because it becomes dominated by either frictional behaviour causing locking in the isolator, or nonlinear hardening of the spring causing the jump up and drop down frequencies to increase.

With some further post processing, the relative displacement of the mass compared to the shaker displacement can be calculated. This is superimposed upon a backbone curve determined using the procedure in Appendix A, which can be calculated with the polynomial coefficients fitted to the static data, in Fig. 15. It can be seen that at low frequencies the locking-up of the device due to friction results in a poor match between response and the backbone curve, particularly in parts (a) and (d) where the lower excitation amplitude is insufficient to break friction at these frequencies. However, when the device is not locked-up, the data follows the backbone curve well. Note, that for the refined configuration, the low amplitude stiffness is slightly negative according to the polynomial fit, and this results in an unusual backbone curve at low amplitude and frequency, as seen in Fig. 15(d) and (e). 
(a)

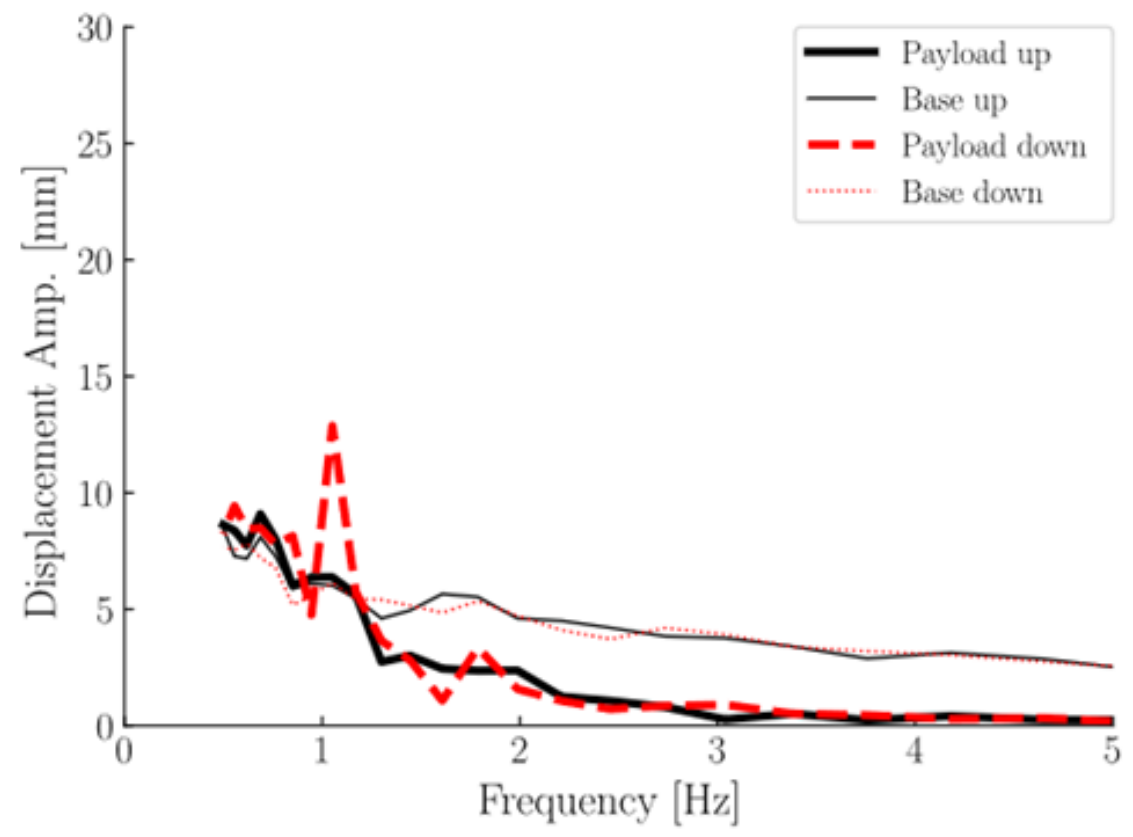

(b)

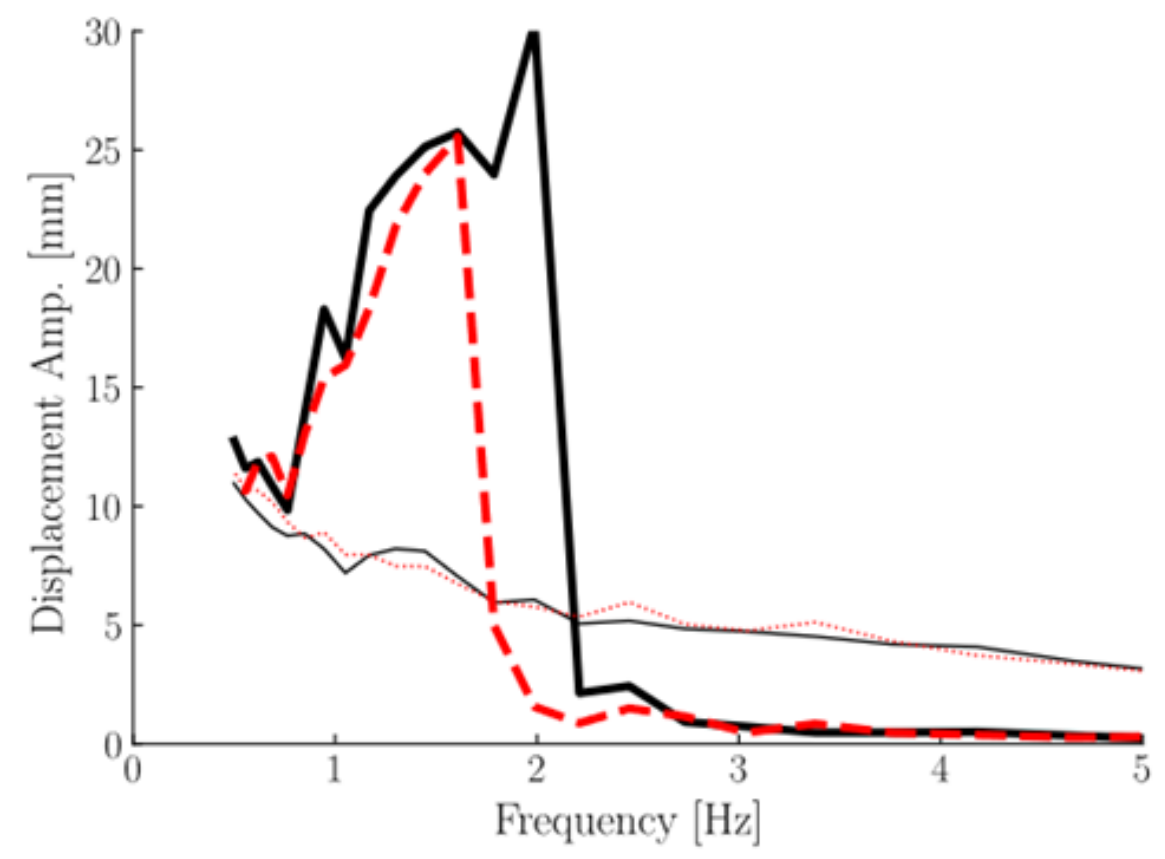

Figure 14. Responses of the refined configuration at voltage amplitude (a) $0.5 \mathrm{~V}$ and (b) $0.6 \mathrm{~V}$. 
(a)

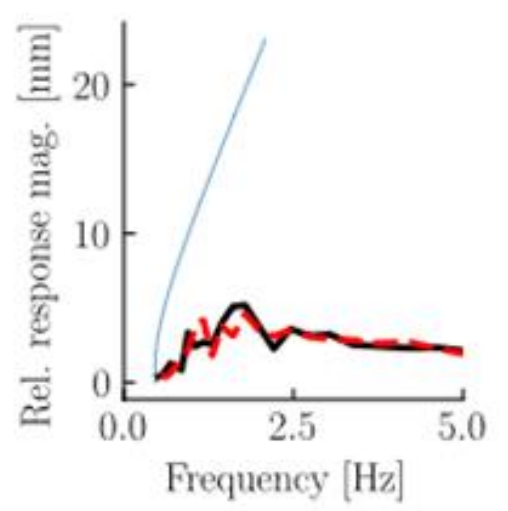

(d)

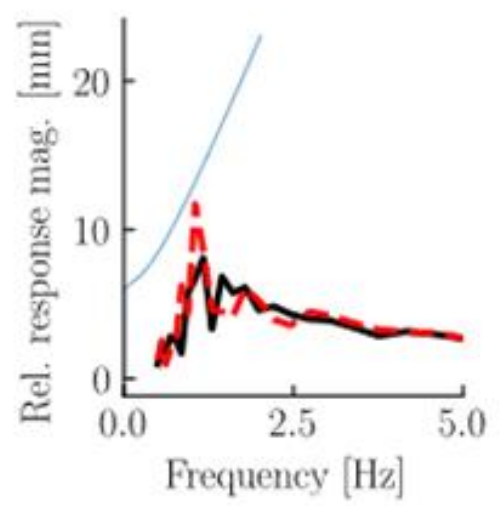

(b)

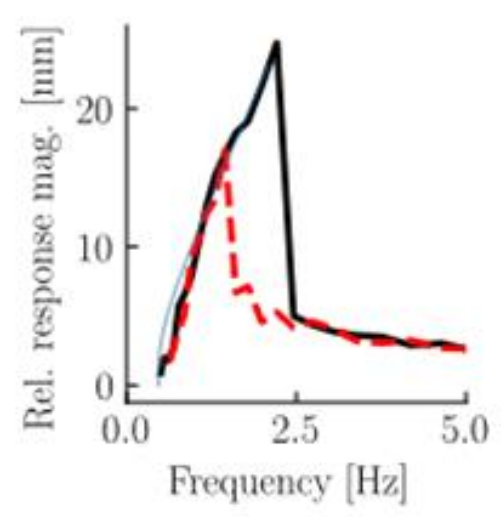

(c)

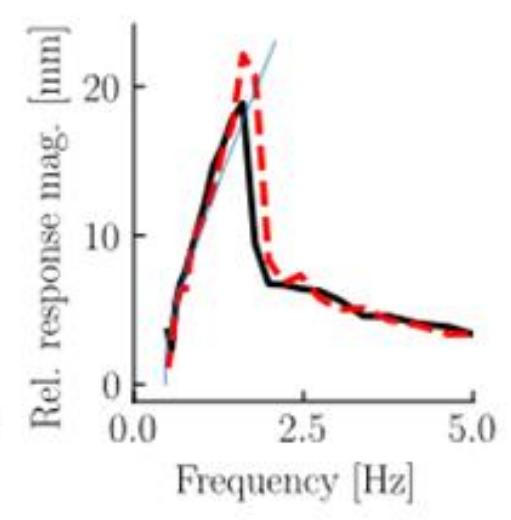

(e)

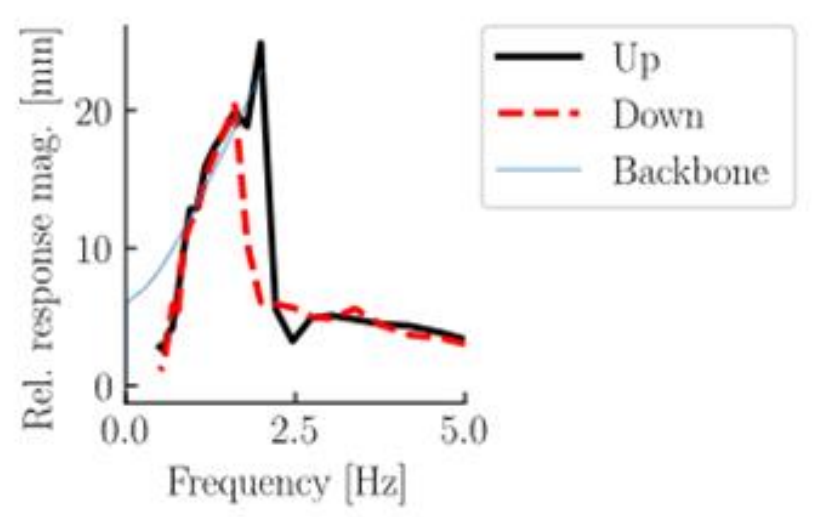

Figure 15. Relative response amplitudes and backbone curves for initial configuration (a) $0.4 \mathrm{~V}$, (b) $0.5 \mathrm{~V}$ and (c) $0.6 \mathrm{~V}$; refined configuration (d) $0.5 \mathrm{~V}$ and (e) $0.6 \mathrm{~V}$.

\section{RESPONSE OF A STEEL BEAM WITH A MASS ATTACHED USING THE QZS DEVICE}

To investigate the efficacy of the QZS device it was used to suspend a $1.5 \mathrm{~kg}$ mass from the tip of a steel box section beam with cross sectional dimensions $100 \mathrm{~mm} \times 50 \mathrm{~mm}$, wall thickness $3 \mathrm{~mm}$, with a free length of $1.8 \mathrm{~m}$. The frequency response function (FRF) of the beam was measured with and without the attached mass. If the QZS device was to perform as desired, the attached mass would have a negligibly small effect on resonant frequencies found in the FRF, although some additional damping would occur. The detail of the proposed experiment is shown in Fig. 16. 


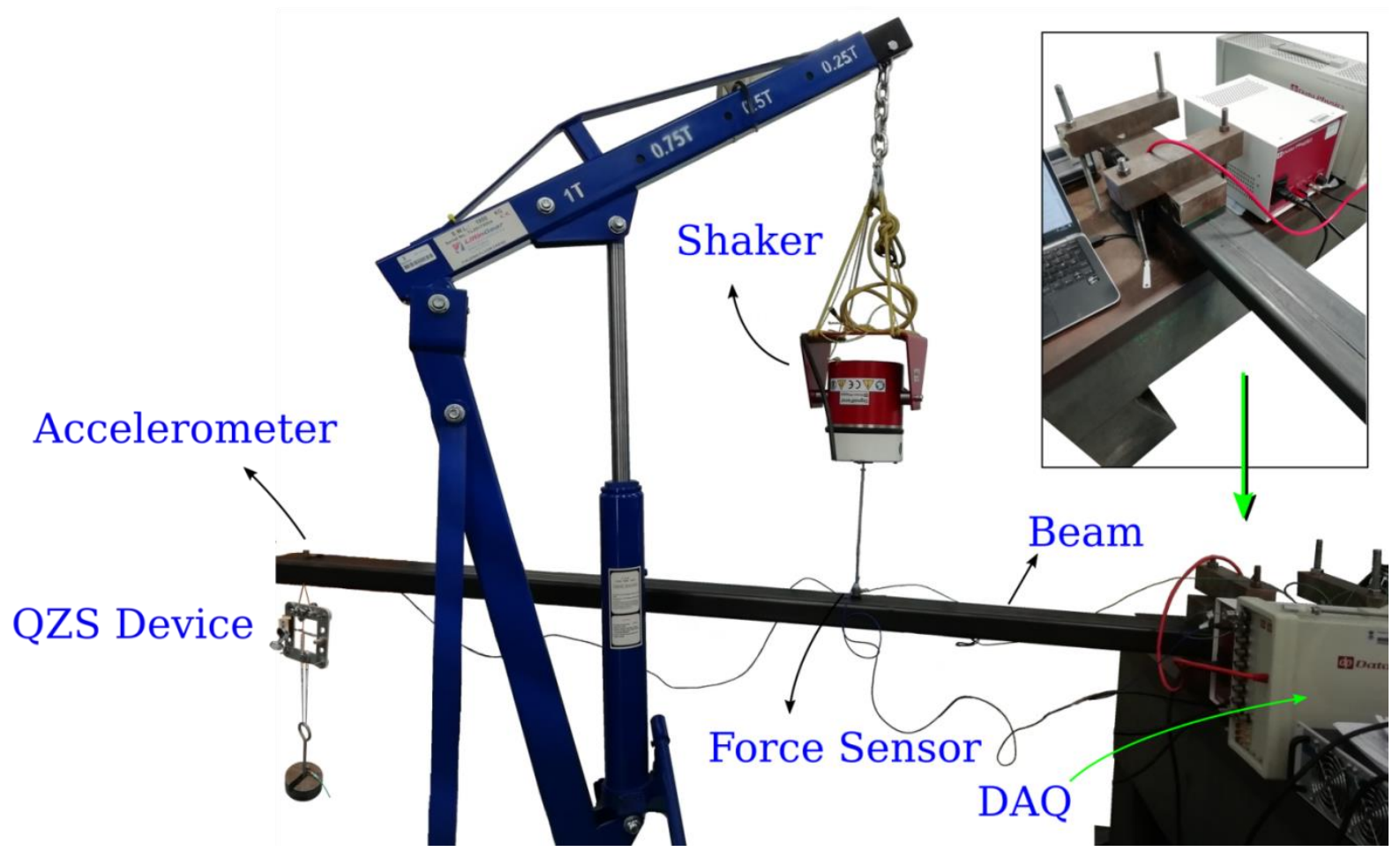

Figure 16. Beam experimental setup showing different views.

The beam was excited by a shaker suspended by elastic cables, attached to the beam at approximately $70 \mathrm{~cm}$ from the fixed end - a location chosen as a compromise between effectively exciting all modes of interest, whilst not demanding excessive stroke from the shaker. The vibration controller and data acquisitions system was a Dataphysics Abaqus 730 signal analyser. A swept sine signal was used to excite the beam over a range of $5-120 \mathrm{~Hz}$ with a logarithmic sweep lasting $40 \mathrm{~s}$. This type of excitation was chosen to ensure that the amplitude of excitation at each frequency was such that no friction locking occurred in the device. Figure 17 shows the various FRFs that were obtained. For the beam, three measurements are shown. One is for the beam alone, the second is when the mass was attached to the tip of the beam using Dyneema ${ }^{\circledR}$ cord, and the third is when the mass was attached via the QZS device. Also shown as grey lines are the FRFs between the applied force and the attached mass. Clearly the use of a broadband FRF characterisation assumes that the structure is behaving linearly, and not showing the complex nonlinear behaviour presented in Section 4.3. By testing in a frequency range significantly beyond the resonance of the isolator, the structure with QZS device responds in a linear-like manner, as confirmed 
by a check on the coherence function which had a minimum value of 0.91 in all tests within the frequency range of interest.

The first comparison to make is between the bare beam response, and that with a mass directly connected to the tip. In this case, it is clear that the mass has a very intrusive effect on the first mode of the beam, shifting the frequency from approximately $15.5 \mathrm{~Hz}$ down to approximately $11.5 \mathrm{~Hz}$. Interestingly, there is clearly some flexibility in the Dyneema® cord because by $60 \mathrm{~Hz}$ the mass is isolated from the beam, and thus has relatively little effect on the $2^{\text {nd }}$ mode. Comparing the response of the beam alone and the beam with the mass attached via the QZS device, shows that the differences are largely corrected through the use of the QZS device to suspend the mass, with both modes showing similar frequencies albeit with more damping in the case of the isolated mass. This highlights the potential of these devices to be used to apply a static load to a structure, with only minor effects on the structure modal properties. It also shows that this configuration applies significant damping to all modes of the structure, in a similar manner to that presented in Figs. 3 and 5.

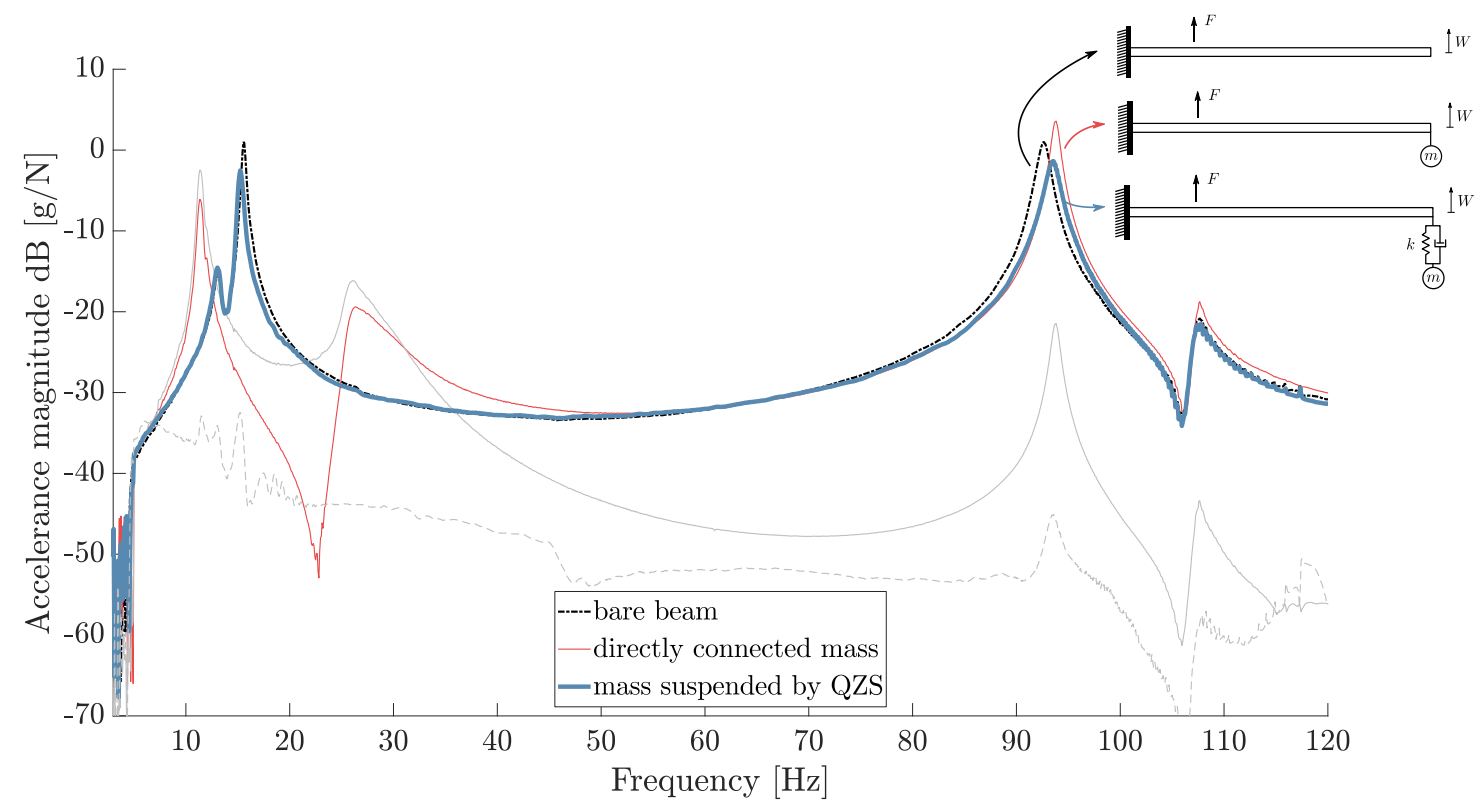

Figure 17. FRF of the bare beam compared to that of beam with a directly connected mass and a mass suspended with the QZS mechanism. Fainted gray lines show that responses of the payload mass were relevant. 


\section{CONCLUSIONS}

In this paper, a mechanically simple device capable of exhibiting quasi-zero stiffness behaviour has been designed, built and tested. The device allows static loads to be applied to a host structure by connecting masses through such devices so that the attached masses do not affect the dynamic behaviour of the loaded structure. The device has a novel design and its stiffness characteristics can be adjusted using two independent mechanisms to (a) adjust the stiffness, and (b) ensure that its nonlinear stiffness is symmetric about its static equilibrium position.

A mathematical description of the device has been presented, and it has been shown experimentally that it can be adjusted to have an extremely low stiffness within the limits of measurement. From a quasi-static test, the main trend of the force-displacement curve showed that it had a cubic stiffness characteristic, and that friction was responsible for its hysteretic behaviour. The dynamic performance of the device was investigated by using it to suspend a mass from a long stroke shaker. Stepped sine tests were used to capture the low frequency resonant behaviour by using a laser displacement sensor. It was shown that, by properly adjusting the device so that it had quasi-zero stiffness, the resonance almost completely disappeared for low levels of excitation, as the device locked up due to friction. The low frequency resonance was dominated by either friction or nonlinear stiffening, and hence a direct measurement of the near zero frequency resonance was not possible. Above the resonance frequency the device acted as an efficient linear isolator.

An experiment was also performed where a mass was suspended on a multi-modal beam structure via the quasi-zero stiffness device. This setup configuration was inspired by a possible application in the aerospace industry, where masses are practically suspended on multi-modal wing structures by soft springs to apply static loads for ground vibration tests, in a way that does not affect modal frequencies of the underlying structures. The main requirement in these cases is to observe the modes of the structure whilst it is subject to static loading, without the static loading affecting the dynamic behaviour of the structure. The results from the experimental tests confirm that the proposed device is able to achieve such a requirement - the lowest resonance frequency of the multi-modal structure was only slighted shifted, despite a suspended mass of about $12 \%$ of that of the host structure. 
FUNDING: A.D. Shaw and M.J. Brennan, would like to acknowledge financial support from the Royal Academy of Engineering [Grant No. DVF1617\613], and B. Tang would like to acknowledge the support of the National Natural Science Foundation of China [Grant No. 11672058].

DECLARATIONS OF INTEREST: none.

\section{REFERENCES}

[1] P. Alabuzhev, A. Gritchin, L. Kim, G. Migirenko, V. Chon, P. Stepanov, Vibration Protecting and Measuring Systems With Quasi-Zero Stiffness. Hemisphere Publishing, New York, 1989.

[2] G. Gatti, M.J. Brennan, B. Tang. Some diverse examples of exploiting the beneficial effects of geometric stiffness nonlinearity, Mechanical Systems and Signal Processing 125 (2019) 4-20.

[3] E.I. Rivin, Passive Vibration Isolation. ASME Press, New York, 2003.

[4] R.A. Ibrahim, Recent advances in nonlinear passive vibration isolators, Journal of Sound and Vibration 314(3-5) (2008) 371-452.

[5] A. Carrella, M.J. Brennan, T.P. Waters, V. Lopes Jr., Force and displacement transmissibility of a nonlinear isolator with high-static-low-dynamic-stiffness, International Journal of Mechanical Sciences, 55(1) (2012) 22-29.

[6] A.D. Shaw, S.A. Neild, D.J. Wagg, Dynamic analysis of high static low dynamic stiffness vibration isolation mounts, Journal of Sound and Vibration, 332(6) (2013) 1437 1455.

[7] Z.Q. Lu, M.J. Brennan, T.J. Yang, X.H. Li, Z.G. Liu, An investigation of a two-stage nonlinear vibration isolation system, Journal of Sound and Vibration 332(6) (2013) 1456-1464.

[8] Z.Q. Lu, M.J. Brennan, H. Ding, L.Q. Chen, High-static-low-dynamic-stiffness vibration isolation enhanced by damping nonlinearity, Science China: Technological Sciences 62(7) (2019) 1103-1110.

[9] Z.Q. Lu, D.H. Gu, H. Ding, W. Lacarbonara, L.Q. Chen, Nonlinear vibration isolation via a circular ring, Mechanical Systems and Signal Processing 136 (2019) 106490. 
[10] Y.S. Zheng, Q.P. Li, B. Yan, Y.J. Luo, X.N. Zhang, A Stewart isolator with high-staticlow-dynamic stiffness struts based on negative stiffness magnetic springs, Journal of Sound and Vibration 422 (2018) 390-408.

[11]Z.Q. Lang, X.J. Jing, S.A. Billings, G.R. Tomlinson, Z.K. Peng, Theoretical study of the effects of nonlinear viscous damping on vibration isolation of sdof systems, Journal of Sound and Vibration 323(1-2) (2009) 352-365.

[12]Z.J. Wu, W.Y. Liu, F.M. Li, C.Z. Zhang, Band-gap property of a novel elastic metamaterial beam with X-shaped local resonators, Mechanical Systems and Signal Processing 134 (2019) 106357.

[13]C. Drezet, N. Kacem, N. Bouhaddi, Design of a nonlinear energy harvester based on high static low dynamic stiffness for low frequency random vibrations, Sensors and Actuators, A: Physical 283 (2018) 54-64.

[14]X. Jiang, D.M. Mcfarland, L.A. Bergman, A.F. Vakakis, Steady state passive nonlinear energy pumping in coupled oscillators: Theoretical and experimental results, Nonlinear Dynamics 33(1) (2003) 87-102.

[15]A.F. Vakakis, O.V. Gendelman, L.A. Bergman, D.M. McFarland, G. Kerschen, Y.S. Lee, Nonlinear Targeted Energy Transfer in Mechanical and Structural Systems Vols. 1 and 2, Springer, Dordrecht, 2009.

[16] O.V. Gendelman, Y. Starosvetsky, M. Feldman, Attractors of harmonically forced linear oscillator with attached nonlinear energy sink I: Description of response regimes, Nonlinear Dynamics 51(1-2) (2008) 31-46.

[17] Y. Starosvetsky, O.V. Gendelman, Attractors of harmonically forced linear oscillator with attached nonlinear energy sink. II: Optimization of a nonlinear vibration absorber. Nonlinear Dynamics 51(1-2) (2008) 47-57.

[18] Y. Starosvetsky, O.V. Gendelman, Vibration absorption in systems with a nonlinear energy sink: nonlinear damping, Journal of Sound and Vibration 324(3-5) (2009) 916939.

[19]R. Viguié, G. Kerschen, Nonlinear vibration absorber coupled to a nonlinear primary system: A tuning methodology, Journal of Sound and Vibration 326(3-5) (2009) 780793.

[20] M.J. Brennan, G. Gatti, The characteristics of a nonlinear vibration neutralizer, Journal of Sound and Vibration 331(13) (2012) 3158-3171. 
[21] J. Yang, Y.P. Xiong, J.T. Xing, Power flow behaviour and dynamic performance of a nonlinear vibration absorber coupled to a nonlinear oscillator, Nonlinear Dynamics 80(3) (2015) 1063-1079.

[22]T. Detroux, G. Habib, L. Masset, G. Kerschen, Performance, robustness and sensitivity analysis of the nonlinear tuned vibration absorber. Mechanical Systems and Signal Processing 60-61 (2015) 799-809.

[23] G. Habib, T. Detroux, R. Viguie, G. Kerschen, Nonlinear generalization of Den Hartog's equal-peak method, Mechanical Systems and Signal Processing 52-53 (2015) 17-28.

[24]B. Tang, M.J. Brennan, G. Gatti, N.S. Ferguson, Experimental characterization of a nonlinear vibration absorber using free vibration, Journal of Sound and Vibration 367 (2016) 159-169.

[25] G. Gatti, Fundamental insight on the performance of a nonlinear tuned mass damper, Meccanica 53(1-2) (2018) 111-123.

[26] W.S. Choi, G.J. Park, Transformation of dynamic loads into equivalent static loads based on modal analysis, International Journal for Numerical Methods in Engineering 46(1) (1999) 29-43.

[27] S.E. Woodard, J.M. Housner, Nonlinear behavior of a passive zero-spring-rate suspension system, Journal of Guidance, Control, and Dynamics, 14(1) (1991) 84-89.

[28]H.A. Lee, Y.I. Kim, G.J. Park, R.M. Kolonay, M. Blair, R.A. Canfield, Structural optimization of a joined wing using equivalent static loads, Journal of Aircraft 44(4) (2007) 1302-1308.

[29] G. Molinari, M. Quack, A.F. Arrieta, M. Morari, P. Ermanni, Design, realization and structural testing of a compliant adaptable wing, Smart Materials and Structures 24(1) (2015) 105027.

[30]D.M. Tang, E.H. Dowell, Experimental and theoretical study of gust response for highaspect-ratio wing, AIAA Journal 40(3) (2002) 419-429.

[31]D.M. Tang, E.H. Dowell, Aerodynamic loading for an airfoil with an oscillating gurney flap, Journal of Aircraft 44(4) (2007) 1245-1257.

[32] S. Keye, Fluid-structure coupled analysis of a transport aircraft and flight-test validation, Journal of Aircraft 48(2) (2011) 381-390.

[33]C.S. Chang, D.H. Hodges, Parametric studies on ground vibration test modeling for highly flexible aircraft, Journal of aircraft 44(6) (2007) 2049-2059.

[34]L.F. Yang, R.C. Tzeng, C.P. Kuo, Adaptive control of a zero-stiffness suspension system, Journal of guidance, control, and dynamics 19(4) (1996), 794-800. 
[35]X.C. Huang, X.T. Liu, J.Y. Sun, Z.Y. Zhang, H.X. Hua, Vibration isolation characteristics of a nonlinear isolator using Euler buckled beam as negative stiffness corrector: a theoretical and experimental study, Journal of Sound and Vibration 333(4) (2014)1132-1148.

[36]X.C. Huang, X.T. Liu, J.Y. Sun, Z.Y. Zhang, H.X. Hua, Effect of the system imperfections on the dynamic response of a high-static-low-dynamic stiffness vibration isolator, Nonlinear Dynamics 76(2) (2014) 1157-1167.

[37]X.C. Huang, X.T. Liu, H.X. Hua, Effects of stiffness and load imperfection on the isolation performance of a high-static-low-dynamic-stiffness non-linear isolator under base displacement excitation, International Journal of Non-Linear Mechanics 65 (2014) $32-43$.

[38] A.D. Shaw, S.A. Neild, M.I. Friswell, Relieving the effect of static load errors in nonlinear vibration isolation mounts through stiffness asymmetries, Journal of Sound and Vibration 339 (2015) 84-98.

[39]B. Yan, H.Y. Ma, L. Zhang, W.G. Zheng, K. Wang, C.Y. Wu, A bistable vibration isolator with nonlinear electromagnetic shunt damping, Mechanical Systems and Signal Processing 136 (2020) 106504.

[40]E. Palomares, A. Nieto, A. Morales, J. Chicharro, and P. Pintado, Numerical and experimental analysis of a vibration isolator equipped with a negative stiffness system, Journal of Sound and Vibration 414 (2018) 31-42.

[41]N. Zhou, K. Liu, A tunable high-static-low-dynamic stiffness vibration isolator, Journal of Sound and Vibration 329(9) (2010) 1254-1273.

[42]T.D. Le, V.A.D. Nguyen, Low frequency vibration isolator with adjustable configurative parameter, International Journal of Mechanical Science 134 (2017) 224-233.

[43]Q. Wang, J.X. Zhou, D.L. Xu, H.J. Ouyang, Design and experimental investigation of ultra-low frequency vibration isolation during neonatal transport, Mechanical Systems and Signal Processing 139 (2020) 106633.

[44] A. Carrella, M.J. Brennan, T.P. Waters, Static analysis of a passive vibration isolator with quasi-zero-stiffness characteristic, Journal of Sound and Vibration, 301(3-5) (2007) 678-689.

[45] Y. Sun, J.S. Zhou, D. Gong, W.J. Sun, Z.H. Xia, Vibration control of high-speed trains self-excitation under-chassis equipment by HSLDS vibration isolators, Journal of Mechanical Science and Technology, 33(1) (2019) 65-76. 
[46]F. Fahy F, J. Walker, Advanced Applications in Acoustics, Noise and Vibration, Taylor \& Francis, London, 2005.

[47]D.J. Mead, Passive Vibration Control. John Wiley \& Sons Ltd, Chichester, 1999.

[48]I. Kovacic, M.J. Brennan, The Duffing Equation: Nonlinear Oscillators and their

Behaviour, John Wiley \& Sons Ltd, Chichester, 2011.

\section{Appendix A: Calculation of backbone curves}

This appendix derives the backbone curves plotted in Figure 15 following an abridged form of the analysis used in [48]. The system comprising the payload mass suspended via the nonlinear isolator from the electro dynamic shaker can be represented by

$$
m \ddot{x}+P(z)=0,
$$

where $x$ is the displacement of the payload mass, $P(z)$ is the restoring force of the isolator given by Eq. (15), and $z=x-r$, is the relative displacement of the mass compared to the motion of the shaker, $r$. Assuming a base excitation of the form $r=R \cos \Omega t$, Eq. (A1) can be written in terms of the relative displacement and the base excitation, to give

$$
m \ddot{z}+P(z)=-m \ddot{r}=m R \Omega^{2} \cos \Omega t .
$$

An underlying conservative system can be extracted from Eq. (A2) as

$$
m \ddot{z}+P(z)=0
$$

where friction has been neglected from $P(z)$, and furthermore $z$ and $P(z)$ have been translated so that the origin is at the static equilibrium position, so that for this calculation $P(z)=p_{1} z+p_{2} z^{2}+p_{3} z^{3}$. Equation (A4) strongly influences the dynamics of Eq. (A3), as it captures all inertial and elastic forces. Its solution gives a relationship between amplitude and frequency known as backbone curve, which gives the trend of the forced response given by Eq. (A2).

The backbone curve is found by assuming Eq. (A4) has a solution of $z=Z \cos \Omega t$. Terms including $\cos \Omega t$ are gathered together and all others are neglected (reasonable for odd or nearly odd $P(z))$ to obtain [48] 


$$
\Omega=\sqrt{\frac{p_{1}+\frac{3 p_{3} Z^{2}}{4}}{m}} .
$$

